\title{
Axonal Growth of Midbrain Dopamine Neurons is Modulated by the Cell Adhesion Molecule ALCAM Through Trans-Heterophilic Interactions with L1cam, Chll, and Semaphorins
}

\author{
@Christopher R. Bye, Valeria Rytova, Walaa F. Alsanie, @Clare L. Parish,* and @Lachlan H. Thompson* \\ Florey Institute for Neuroscience and Mental Health, The University of Melbourne, Royal Parade, Parkville, Victoria, Australia 3010
}

\begin{abstract}
The growth of axons corresponding to different neuronal subtypes is governed by unique expression profiles of molecules on the growth cone. These molecules respond to extracellular cues either locally though cell adhesion interactions or over long distances through diffusible gradients. Here, we report that that the cell adhesion molecule ALCAM (CD166) can act as an extracellular substrate to selectively promote the growth of murine midbrain dopamine $(\mathrm{mDA})$ neuron axons through a trans-heterophilic interaction with mDA-bound adhesion molecules. In mixed-sex primary midbrain cultures, the growth-promoting effect of ALCAM was abolished by neutralizing antibodies for components of the Semaphorin receptor complex Nrp1, Chl1, or L1 cam. The ALCAM substrate was also found to modulate the response of $\mathrm{mDA}$ neurites to soluble semaphorins in a context-specific manner by abolishing the growth-promoting effect of Sema3A but inducing a branching response in the presence of Sema3C. These findings identify a previously unrecognized guidance mechanism whereby cell adhesion molecules act in trans to modulate the response of axonal growth cones to soluble gradients to selectively orchestrate the growth and guidance of mDA neurons.
\end{abstract}

Key words: CAMs; connectivity; guidance; mesencephalon; Nrp1

\section{Significance Statement}

The mechanisms governing the axonal connectivity of midbrain dopamine ( $\mathrm{mDA}$ ) neurons during neural development have remained rather poorly understood relative to other model systems for axonal growth and guidance. Here, we report a series of novel interactions between proteins previously not identified in the context of mDA neuronal growth. Significantly, the results suggest a previously unrecognized mechanism involving the convergence in signaling between local, adhesion and long-distance, soluble cues. A better understanding of the molecules and mechanisms involved in establishment of the mDA system is important as a part of ongoing efforts to understand the consequence of conditions that may result from aberrant connectivity and also for cell replacement strategies for Parkinson's disease.

\section{Introduction}

The navigation of axons to their correct targets during development is critical for the establishment of the complex neuronal

\footnotetext{
Received Feb. 5, 2019; revised May 21, 2019; accepted July 3, 2019.

Author contributions: C.R.B., C.L.P., and L.H.T. designed research; C.R.B., V.R., and W.F.A. performed research; C.R.B., W.F.A., C.L.P., and L.H.T. analyzed data; C.R.B., C.L.P., and L.H.T. wrote the paper; C.L.P. and L.H.T. edited the paper.

This work was supported by the National Health and Medical Research Council (NHMRC Grants 1042584 and 1022637). The Florey Institute of Neuroscience and Mental Health acknowledges the strong support from the Victorian Government and in particular the funding from an Operational Infrastructure Support Grant. C.R.B. is supported by an NHMRC Peter Doherty training fellowship, C.L.P. is an NHMRC Senior Research Fellow. We thank Jason Howitt for technical assistance with PLA Assays, Mong Tien and Jessica Kauhausen for assistance with tissue processing and histology, and Richard Anderson and John Hemperly for provision of the $6096 \mathrm{~L} 1$ blocking antibody.

The authors declare no competing financial interests.

*C.L.P. and L.H.T. contributed equally to this work.
}

circuitry required for normal function of the CNS. Some model systems have been studied more extensively than others; for example, midline crossing of commissural axons and establishment of the retinotectal and thalamocortical systems (for recent reviews, see(Evans and Bashaw, 2010; Garel and López-Bendito, 2014; Herrera et al., 2019). These findings have helped to identify fundamental mechanisms and signaling molecules that allow the highly specific navigation of ax-

Correspondence should be addressed to Christopher R. Bye at chris.bye@florey.edu.au or Lachlan H. Thompson at lachlant@unimelb.edu.au.

W. F. Alsanie's present address: Department of Clinical Laboratories, The Faculty of Applied Medical Sciences, Taif University, Taif, Saudi Arabia.

https://doi.org/10.1523/JNEUROSCI.0278-19.2019

Copyright $\odot 2019$ the authors 
ons to predefined targets through a dynamic and complicated extracellular environment. Soluble guidance cues, including the four classical families comprising Semaphorins, Ephrins, Netrins, and Slits, as well as more recent morphogens such as FGFs, Hedgehogs, and Wnts, have largely been considered as the primary determinant in mediating axon growth and connectivity (Pasterkamp and Kolodkin, 2013; Van Battum et al., 2015). Although the combinatorial presentation of soluble cues and their corresponding ligands allows for remarkable diversity in context-specific axonal responses, they are not in themselves sufficient to facilitate the enormous range of independent neuronal pathways simultaneously formed during neural development.

Cell adhesion molecules (CAMs), including Igs, cadherins, integrins, and neurexins/neuroligins, are increasingly being recognized as important determinants in the regulation of axon guidance (Walsh and Doherty, 1997). The Ig-superfamily (IgSFCAMs) is the largest and most studied family of CAMs in neural development, regulating axon growth, pathway formation, and synaptogenesis through contact-mediated adhesion with themselves (homophilic) or other CAMs (heterophilic) (Pollerberg et al., 2013). Previously considered mutually separated events, with diffusible gradients providing long-range signaling cues for axons to traverse across extended distances and membrane-associated molecules acting as short-range "detectors" between the axon tip and surrounding extracellular matrix, more recent studies have begun to recognize direct interactions between CAMS and the classical axon guidance/morphogen receptors (Demyanenko et al., 2011; Wade et al., 2012).

Here, we report the convergence of signaling through both cell adhesion and soluble growth cues as a novel mechanism regulating the growth of midbrain dopamine $(\mathrm{mDA})$ neurons. These neurons form the nigrostriatal, mesolimbic, and mesocortical pathways, projecting from the ventral midbrain to targets in the striatum and cortex, and are essential for the control of voluntary movement, as well as impulse control and emotion. Current models of mDA axonal growth are based almost entirely on signaling through soluble guidance cues (Prasad and Pasterkamp, 2009; Prestoz et al., 2012) and disruption in expression of these molecules may underlie neurodevelopmental deficits in the establishment of mDA circuitry. Dysfunction of mDA circuitry is associated with a range of neurodegenerative and neuropsychiatric conditions including Parkinson's disease, drug addiction, depression, and schizophrenia (Van den Heuvel and Pasterkamp, 2008).

In a recent study incorporating genetic screening of the developing mDA system, we identified the IgSF-CAM family members Alcam (activated leukocyte cell adhesion molecule; CD166) and Chl1 (close homolog of L1) during the peak period of axon growth in dopamine pathways (Bye et al., 2015). Here, we show that both are expressed in the developing $\mathrm{mDA}$ pathway and use a series of functional in vitro assays to demonstrate a novel role for Alcam as a selective $\mathrm{mDA}$ axonal growth-promoting substrate through the formation of heterodimeric interactions with $\mathrm{mDA}$ neuron-bound Chl1 or L1cam. Furthermore, we show that Alcam can modulate the response of growing $\mathrm{mDA}$ axons to soluble semaphorins via interactions with the Neuropilin1 receptor. These findings not only identify new molecules for establishment of mDA circuitry, but also highlight the capacity for adhesionbased and soluble signaling mechanisms to converge to provide a potentially vast diversity of context-dependent functional responses to individual signaling pathways.

\section{Materials and Methods}

Ethical approval and animal housing. The use of animals in this study conformed to the Australian National Health and Medical Research Council's published Code of Practice for the Use of Animals in Research and was approved by the Florey Neuroscience Institute animal ethics committee. Alcam ${ }^{\text {tm } 1 \text { Jawe }}$ mice were from The Jackson Laboratory (stock \#010635) (Weiner et al., 2004). All animals were housed under a $12 \mathrm{~h}$ light/dark cycle with ad libitum access to food and water.

Surgical procedures and graft quantification. Groups of sex-balanced Alcam $^{-1-}$ and littermate controls received unilateral 6-hydroxydopamine lesions of the midbrain DA system followed by transplantation of embryonic day 12.5 (E12.5) TH-GFP ventral midbrain cell suspension into the striatum (100,000 cells per mouse) as described previously (Bye et al., 2012). Quantification of graft neuron number, innervation volume and density were conducted 6 weeks after transplantation as described previously (Bye et al., 2015).

Primary culture axon growth assays. Microdissection and dissociation of mixed-sex mouse ventral midbrain was conducted on time-mated E12.5 C57BL/6 or Alcam ${ }^{-1-}$ mice. Mice were time-mated overnight and E0.5 was designated after visualization of a vaginal plug the next morning. Ventral midbrain microdissection was performed as described previously (Thompson and Parish, 2013) in chilled L15 medium and incubated in HBSS containing 0.05\% trypsin and $0.1 \%$ DNase for $15 \mathrm{~min}$ at $37^{\circ} \mathrm{C}$ (all from Life Technologies). Tissue pieces were washed 3 times in HBSS and resuspended in a serum-free minimal N2 media consisting of a 1:1 F12/MEM supplemented with 15 mM HEPES buffer, $1 \mathrm{~mm}$ glutamine, $6 \mathrm{mg} / \mathrm{ml}$ glucose, $1.5 \mathrm{mg} / \mathrm{ml}$ AlbuMAX, and N2 supplement (all from Life Technologies). The tissue pieces were dissociated to a singlecell suspension, cell viability and density determined by trypan blue exclusion counting, and seeded in 48-well plates at a density of 125,000 cells per well. Cells were cultured in vitro at $37^{\circ} \mathrm{C}$ in $3 \% \mathrm{O}_{2}$ and $5 \% \mathrm{CO}_{2}$ for $72 \mathrm{~h}$ for axon growth assays or for $36 \mathrm{~h}$ for proximity ligation assay (PLA) and immunohistochemistry.

Plates were prepared before cell seeding by coating sequentially with PDL (10 $\mu \mathrm{g} / \mathrm{ml}$; Sigma-Aldrich) for $12 \mathrm{~h}$, goat anti-human-IG F $\left(\mathrm{ab}^{\prime}\right)_{2}$ ( $35 \mu \mathrm{g} / \mathrm{ml}$; Jackson ImmunoResearch) for $12 \mathrm{~h}$, blocked in 1\% BSA for 30 min, followed by addition of Alcam recombinant Fc Chimera protein (100 ng/ml; R\&D Systems) for $12 \mathrm{~h}$. The anti-human-IgG secondary antibody provided alignment and high-density clustering of Alcam through binding to the human IgG located at the C terminus of the Alcam Chimera protein (Buhusi et al., 2009; Thelen et al., 2012). Wells were washed three times with PBS after the PDL-, anti-human-IG-, and Alcam-coating steps. PDL and anti-human-IG controls were prepared in parallel using PBS to replace the anti-human-IG and/or Alcam coating. For each experiment, effects were normalized to PDL-only coated reference wells (mean normalized control value set at 100\%). Function blocking antibodies and Semaphorin were added $2 \mathrm{~h}$ after seeding as follows: rat anti-L1cam antibody $(0.1 \mu \mathrm{g} / \mathrm{ml}$; Millipore), rabbit anti-L1 blocking antibody $6096(10 \mu \mathrm{g} / \mathrm{ml}$; BD Technologies) goat anti-Chl1 blocking antibody ( $1 \mu \mathrm{g} / \mathrm{ml}$; R\&D Systems), goat anti-Nrp1 (2 $\mu \mathrm{g} / \mathrm{ml}$; R\&D Systems), goat anti-Nrp2 (2 $\mu \mathrm{g} / \mathrm{ml}$; R\&D Systems), purified rat IG control $(0.1 \mu \mathrm{g} / \mathrm{ml}$; Jackson ImmunoResearch), purified rabbit IG control (10 $\mu \mathrm{g} / \mathrm{ml}$; Jackson ImmunoResearch), purified goat IG control $(1 \mu \mathrm{g} / \mathrm{ml}$; Jackson ImmunoResearch), Sema3A (100 ng/ml; R\&D Systems), Sema3C (100 ng/ml; R\&D Systems), and Sema3F (100 ng/ml; R\&D Systems). Neuronal cell counts and neurite morphology were analyzed as described previously (Blakely et al., 2011).

Tissue processing and immunohistochemistry. Embryonic and adult tissue was processed as described previously in detail (Bye et al., 2015). Primary antibodies used in immunohistochemistry were as follows; goat anti-Alcam (1:800; R\&D Systems), mouse anti- $\beta$ III-tubulin (1:1000; Promega), rat anti-Chl1 (1:600; Millipore), goat anti-Chl1 (1:800; R\&D Systems), rabbit anti-GFP (1:20,000; Abcam), rat anti-L1cam (1:1000; Millipore), F-actin phalloidin iFluor 555 reagent (1:1000; Abcam), rabbit anti-Nrp1 (1:500; Abcam), goat anti-Nrp2 (1:200; R\&D Systems), chicken anti-TH (1:400; Abcam), mouse anti-TH (1:300; Millipore), rabbit anti-TH (1:800; PelFreez), and sheep anti-TH (1:600; PelFreez). 
High-magnification images were captured on a Zeiss Pascal confocal microscope system.

$q R T-P C R$. RNA was isolated from embryonic mouse ventral midbrain using a RNeasy Micro kit (Qiagen) and reverse transcribed using a SuperScript VILO cDNA Synthesis Kit (Invitrogen). qRT-PCR was performed using a SYBR GreenER qPCR SuperMix Universal (Invitrogen) on a Rotor-Gene 6000 thermocycler (Qiagen) and analyzed using the comparative $\Delta \Delta \mathrm{CT}$ method. Primer sequences were as follows: Mthfd1 forward, 5' -AAGGAAAGTCGTGGGTGATG-3', Mthfd1 reverse, 5'-T ATGTCCCCCGTTGACTGAT-3'; Alcam forward, 5'-TTCAGGAGGT TGAGGGACTG-3', Alcam reverse, 5'-CAGTCCACTGGGGTCAG TTT-3'.

Embryonic quantification. Ventral mDA neuron number, pathway volume, and the density of varicosities reaching the ganglionic eminence were quantified in Alcam ${ }^{-1-}$ and littermate controls at E14.5 as described previously (Blakely et al., 2011).

Proximity ligation assay. The Duolink Proximity Ligation Assay (Sigma-Aldrich) was performed as per the manufacturer's instructions with the following modifications. PLA probes were diluted 1:20 and ligation and amplification reagents were diluted 1:2. Goat anti-Alcam (1: 500; R\&D Systems) and rabbit anti-Nrp1 (1:500; Abcam) were indirectly conjugated with PLA probes and rat anti-Chl1 ( $1 \mu \mathrm{g} / \mathrm{ml}$; Millipore $)$ was directly conjugated using the Duolink In Situ Probemaker Kit and diluted 1:200. Images were captured at $40 \times$ using the Zeiss Pascal confocal microscope system and positive puncta were counted in the growth cone of four biological replicates $\left(20 \mathrm{TH}^{+}\right.$neurons per replicate) and expressed as density (puncta per growth cone area).

Statistical analysis. All quantitative data are represented as mean \pm SEM and were analyzed with Prism 8 software (GraphPad). Comparison of values for statistical significance between groups was performed using Student's $t$ test for comparison of two data points, and one-way ANOVA or two-way ANOVA where appropriate. Tukey's multiple-comparisons test was conducted for all comparisons for one-way ANOVA. Sidak's multiple-comparisons test was conducted comparing the control substrate group with the Alcam substrate group (the primary comparison in this study) for each factor unless an alternative comparison was specified.

\section{Results}

\section{Alcam is transiently expressed along the midbrain} dopaminergic axonal pathway during development

Immunohistochemistry in embryonic mice at E12.5 showed that Alcam was prominently expressed in discrete locations throughout the developing brain, notably the ventral midbrain, hypothalamus, and throughout the cortex (Fig. 1A). Alcam was strongly expressed in the midbrain (Fig. $1 B, C$ ) and throughout the developing medial forebrain bundle, most notably within the domain containing rostrally projecting $\mathrm{TH}^{+} \mathrm{mDA}$ neurons (Fig. $1 D, E$ ). High-magnification images suggested expression of Alcam on $\mathrm{mDA}$ axons, with broad Alcam expression additionally located on surrounding non-mDA axons and cell bodies along the pathway (Fig. $1 F$ ). The intensity of Alcam labeling progressively decreased rostrally in $\mathrm{mDA}$ axons projecting along the medial forebrain bundle, and was largely absent in the ventral forebrain, including in the ganglionic eminence (GE) (Fig. 1G,H).

By E14.5, following the arrival of a majority of mDA axons in the forebrain and the initial innervation of the GE (Van den Heuvel and Pasterkamp, 2008), Alcam gene expression was significantly reduced in the ventral midbrain by $74.8 \pm 0.1 \%$ in E14.5 compared with E10.5 and $64.3 \pm 0.1 \%$ in E14.5 compared with E12.5 (Fig. 1I). A reduced intensity of immunolabeled protein expression was also detected in the midbrain at E14.5 (Fig. $1 J, K$ ), throughout the mDA pathway (Fig. $1 L, M$ ), and in the forebrain $\mathrm{GE}$ terminal area (Fig. $1 \mathrm{~N}, \mathrm{O}$ ). Interestingly, Alcam was strongly expressed in the developing cortex immediately adjacent to the GE and high-magnification imaging showed presumptive mesocortical mDA axons in close apposition to the strongly
Alcam-expressing fibers, apparently wrapping around the larger Alcam ${ }^{+}$fiber bundles (Fig. 1P). A similar pattern was seen for the mDA mesohabenular pathway with $\mathrm{TH}^{+}$axons exiting the midbrain in close apposition with strongly Alcam-expressing fiber bundles (Fig. 1Q,R). Areas of high Alcam expression were also observed in discrete nuclei adjacent to the mDA pathway, including the developing hypothalamus (Fig. $1 L$ ).

In the adult brain, Alcam expression was completely absent in $\mathrm{mDA}$ neurons and axons (Fig. 1S,T), but striking in specificity of expression almost exclusively in medium spiny projection neurons within the striatum (Fig. $1 U$ ), including their axonal projections (Fig. $1 \mathrm{~V}$ ) and terminal fields in the globus pallidus and substantia nigra (Fig. $1 W$ ).

\section{Alcam substrate promotes midbrain dopamine axonal growth in vitro}

The effect of extracellular Alcam on mDA neurite growth was assessed using primary mouse cultures isolated from the ventral midbrain and plated onto Alcam-coated culture wells. Immunocytochemistry in primary midbrain cultures showed that Alcam was strongly expressed on $\mathrm{TH}^{+} \mathrm{mDA}$ neurons, with variable expression across non-mDA neurons (Fig. $2 A$ ). The pattern of expression was uniform regardless of cell type, appearing as distinctly punctate and distributed throughout the cell soma and all neurites (Fig. $2 B, C$ ), with high-magnification images illustrating prominent expression in mDA growth cones (Fig. 2D,E).

Four parameters of neurite initiation and extension were quantified for dopaminergic $\left(\mathrm{TH}^{+}\right)$neurons grown on wells coated with IgG control (Fig. $2 F$ ) or high-density Alcam substrate (Fig. $2 G$ ). Alcam substrate resulted in a $67.6 \pm 14.9 \%$ increase in the length of the dominant neurite (the longest, most evident neurite arising from the soma, and thereby presumptive axon) (Fig. $2 \mathrm{H}$ ) and a $62.8 \pm 23.6 \%$ increase in cumulative neurite length per neuron (Fig. 2I). No significant effect was observed on the number of neurite branches or the number of neurites per neuron (Fig. $2 J, K$ ), although the number of branches per neuron showed a consistent trend to increase in the presence of the Alcam substrate across a number of analyses. Total neurite length was found to consistently parallel dominate neurite length, and neurite number showed no significantly difference, so only dominant neurite length and branch number are subsequently reported. To determine the specificity of substratebased Alcam signaling for $\mathrm{mDA}$ neurons, the same growth parameters were measured for non- $\mathrm{mDA}\left(\mathrm{TH}^{-} / \mathrm{Tuj}^{+}\right)$neurons in the same cultures (Fig. $2 L, M$ ). Non-mDA neurons showed no change in the neurite length or branch number when grown on an Alcam substrate (Fig. 2N,O).

To examine the effect of cell-intrinsic loss of Alcam function on neurite growth in vitro, primary midbrain cultures from WT and Alcam ${ }^{-1-}$ mice were grown in the absence of an Alcam substrate (Fig. $2 P, Q$ ). No alteration in the neurite length or number of neurite branches per $\mathrm{mDA}$ neuron was observed in Alcam $^{-1-}$ relative to WT neurons (Fig. $2 R, S$ ).

\section{Loss of Alcam function in vivo does not produce a developmental phenotype but impairs growth of transplanted mDA neurons}

To determine the consequence of Alcam loss of function in vivo, the developing mDA pathway was examined in WT and Al$\mathrm{cam}^{-1-}$ mice. There was no significant difference in the number of $\mathrm{TH}^{+} \mathrm{mDA}$ neurons in WT and Alcam ${ }^{-1-}$ mice at E14.5 (Fig. $3 A-C)$ or in adulthood (data not shown). At E14.5, no gross defect in the mDA pathway growth or trajectory was observed 

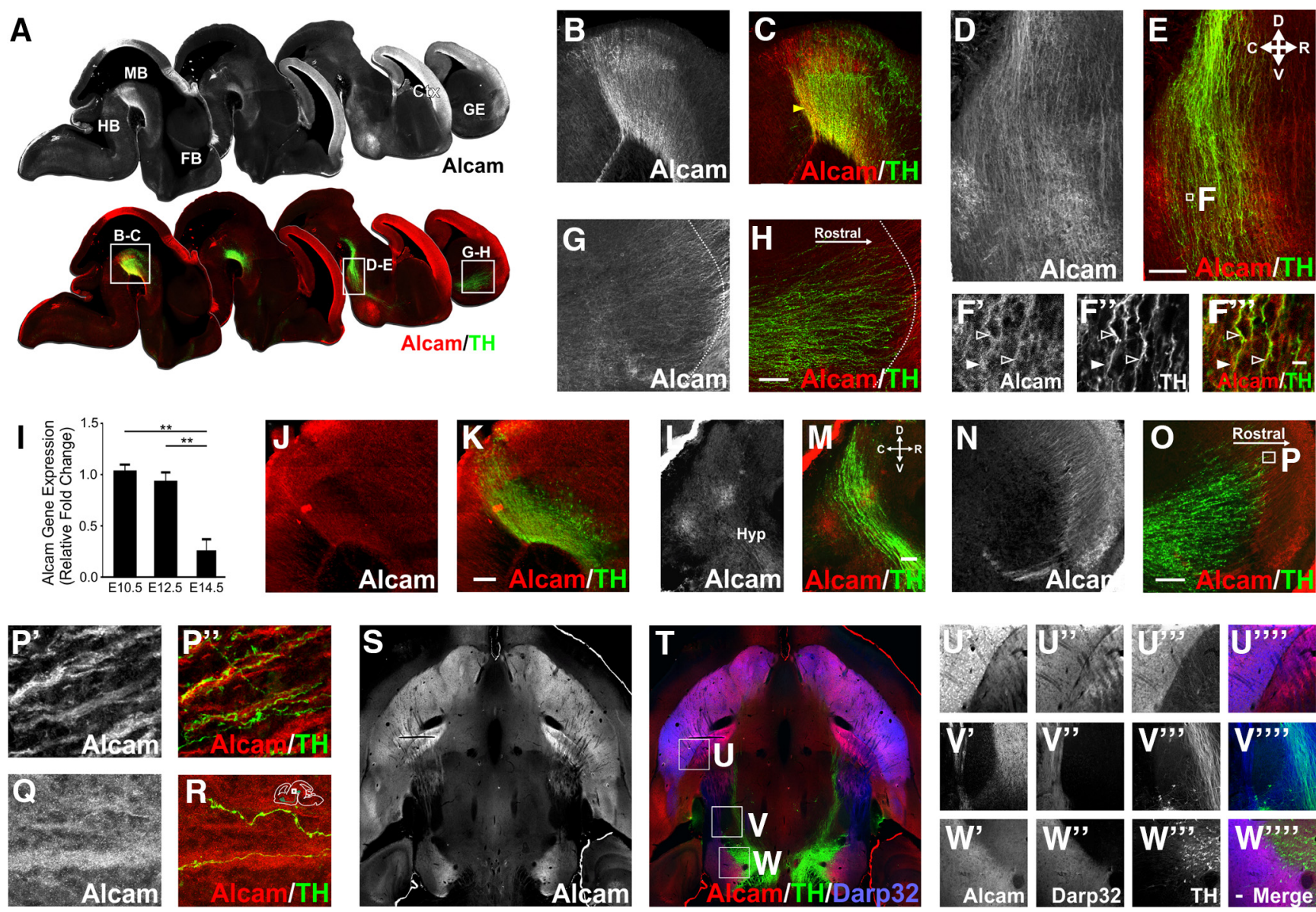

Figure 1. Alcam in vivo expression during formation of the midbrain dopaminergic axonal pathways. In vivo representative sagittal images of whole embryonic brain at E12.5 $(\boldsymbol{A})$ immunolabeled for Alcam and Alcam/TH showing Alcam expression relative to the developing dopaminergic pathway (displayed in a medial to lateral series). Inserts show high Alcam intensity in the ventral midbrain $(\boldsymbol{B}, \boldsymbol{C})$, including in dopamine neurons, and along the axonal pathway $(\boldsymbol{D}, \boldsymbol{E})$. Magnified high-resolution images show the expression of Alcam in both dopaminergic and nondopaminergic axons and cells along the pathway $(\boldsymbol{F})$. Filled arrows indicate Alcam as a substrate expressed along the pathway and empty arrows indicate Alcam expression in $\mathrm{TH}^{+}$axons. In the forebrain $(\boldsymbol{G}, \boldsymbol{H})$, lower Alcam intensity was observed in the GE, with higher intensity observed in nondopaminergic fibers at the boundary of the GE and cortex. Quantification of Alcam gene expression by qRT-PCR (I) showed a significant difference (ANOVA, $F_{(2,7)}=17.02, p=0.0021$ ), with reduced expression at E14.5 compared with E10.5 (ANOVA, $t=5.263, \mathrm{df}=7, p=0.0029, n=4$ ) and E12.5 levels (ANOVA, $t=4.528, \mathrm{df}=7, p=0.0066, n=4)$. AtE14.5, Alcam protein expression was restricted to a caudomedial subpopulation of dopaminergic neurons in the ventral midbrain $(J, K$ ) and largely absent in dopaminergic and nondopaminergic axons along the pathway $(L, M)$ and in the $G E(N, O)$. Strong Alcam intensity continued in nondopaminergic fibers at the boundary of the $G E$ and cortex, magnified images show mesocortical dopaminergic axons wrapping around strongly expressing Alcam fibers descending from the developing cortex into the GE $(\boldsymbol{P})$. Dopaminergic axons were also observed wrapping around intensely Alcam-labeled fibers in the mesohabenular pathway exiting the midbrain $(\boldsymbol{Q}, \boldsymbol{R})$, schematic of image location shown at top right. Horizontal image of whole adult brain $(S, T)$ immunolabeled for Alcam/TH/Darp32 showing no Alcam expression in the fully formed mDA pathway. Inserts show Alcam was expressed intensely in Darp32 ${ }^{+}$medium spiny neurons and axons, not in dopaminergic neurons and axons in the striatum $(\boldsymbol{U})$, along the dopaminergic axonal pathways $(\boldsymbol{V})$, and in the ventral midbrain $(\boldsymbol{W})$. Scale bars: $\boldsymbol{B}-\boldsymbol{E}, 20 \mu \mathrm{m} ; \boldsymbol{F}, 10 \mu \mathrm{m}$; $\mathbf{G}, \boldsymbol{H}, \mathbf{J}-\mathbf{O}, \boldsymbol{U}, \boldsymbol{W}, 20 \mu \mathrm{m}$. Data are shown as mean $\pm \mathrm{SEM}$. Ctx; Cortex; FB, forebrain; GE, ganglionic eminence; HB, hindbrain; Hyp, hypothalamus; $M B$, midbrain. ${ }^{* *} p<0.01$.

(Fig. 3D,E). Quantification of the MFB area (Fig. 3F) measured midway between the $\mathrm{mDA}$ neurons and $\mathrm{GE}$ border (depicted in Fig. $3 G, H$ ), as well as density of neurites (Fig. 3I), showed no significant difference in fasciculation or the number of axons reaching the forebrain.

Compensatory mechanisms are common in loss-of-function studies in developmental neurobiology. To attempt to circumvent this to isolate the effect of loss of Alcam as an extracellular substrate in vivo while retaining intrinsic expression in $\mathrm{mDA}$ neurons (analogous to the in vitro experiments in Fig. 2), we transplanted fetal (E12.5) midbrain from WT mice into adult WT and Alcam $^{-1-}$ mice. Figure $3, J$ and $K$, shows robust Alcam protein expression in the striatum of adult WT mice and complete absence in the brains of Alcam ${ }^{-1-}$ mice. Six weeks after transplantation, immunohistochemistry showed grafts of similar size and with no significant difference in the number of surviving $\mathrm{mDA}$ neurons (WT $1444 \pm 261$; Alcam $\left.^{-1-} 1514 \pm 545\right)$ (Fig. 3L-N). Notably, however, the grafts placed in the Alcam ${ }^{-1-}$ hosts pro- vided significantly less volume and density of innervation of the surrounding host striatum compared with grafts into WT (Fig. $3 O-R)$.

Alcam substrate promotes axon growth through a heterophilic interaction.

We next explored possible mechanisms underlying regulation of $\mathrm{mDA}$ axon growth by Alcam as an extracellular substrate. In both neuronal and non-neuronal contexts, Alcam has been shown to act predominantly through trans (between the growth cone and a substrate), homophilic (self-self) interactions (Tanaka et al., 1991; van Kempen et al., 2001). To determine whether Alcam similarly regulated $\mathrm{mDA}$ axon growth through a trans-homophilic interaction, primary ventral midbrain cultures from WT and Al$\mathrm{cam}^{-1-}$ mice were grown in the presence or absence of Alcam substrate (Fig. 4A,B). Interestingly, loss of function of Alcam in $\mathrm{mDA}$ neurons grown on the Alcam substrate was found to have no effect on the growth-promoting effect of Alcam substrate, 

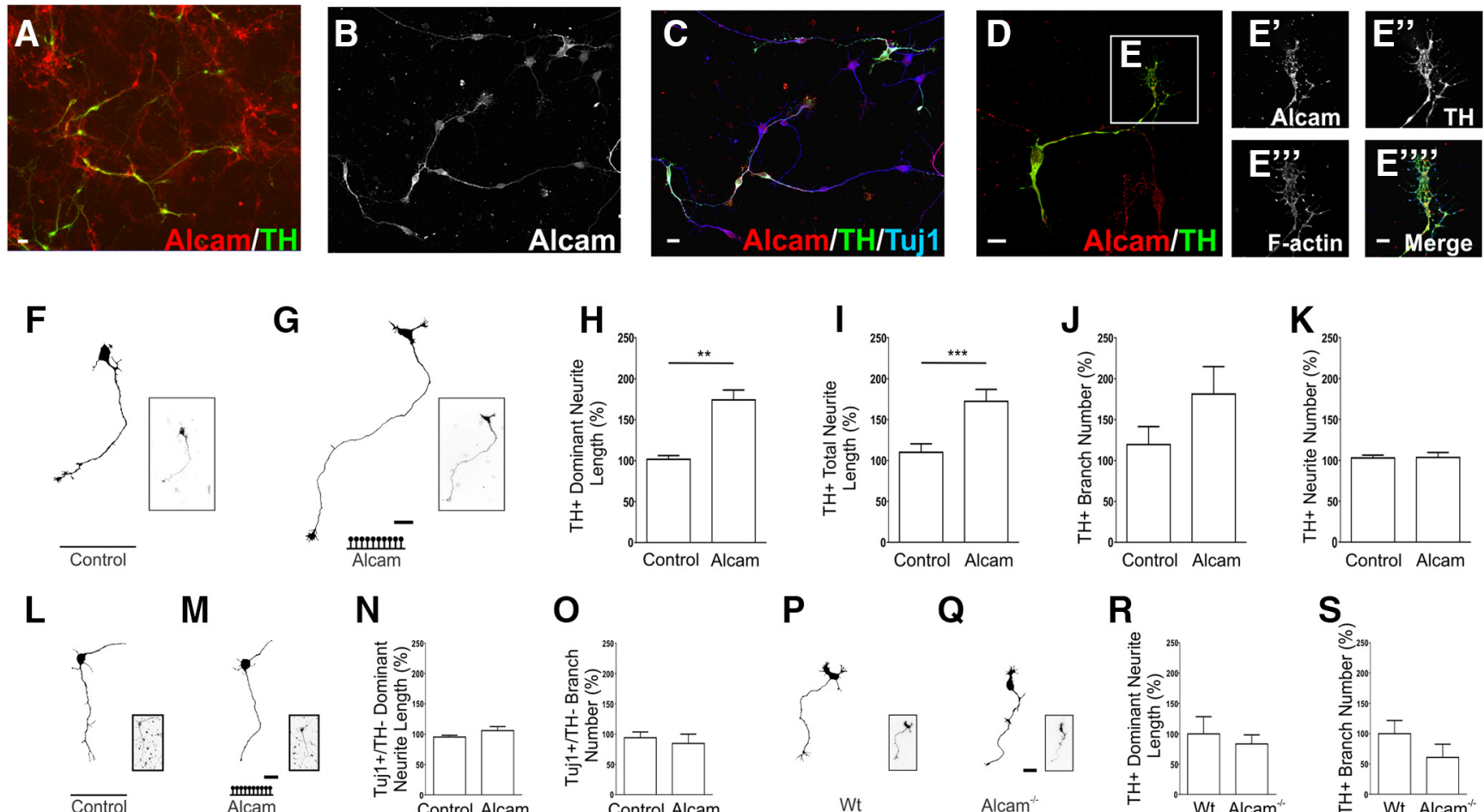

M
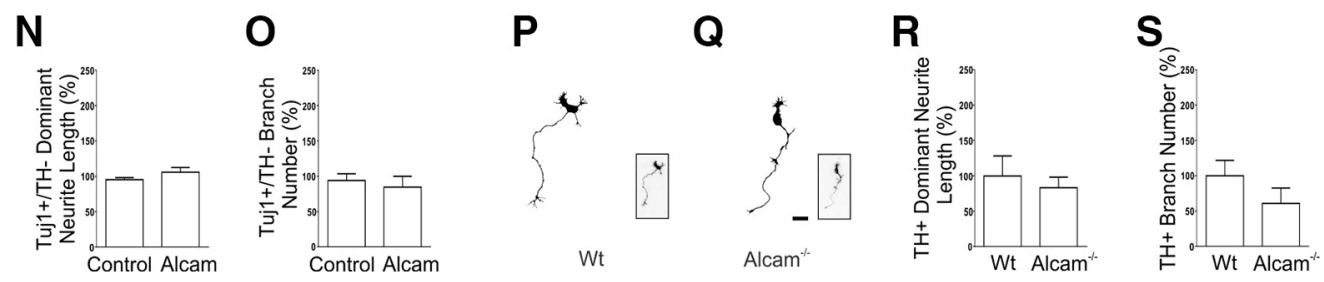

Figure 2. Alcam in vitro expression and function during dopaminergic axonal growth. Representative images of neurons in primary midbrain cultures immunolabeled for Alcam $(\boldsymbol{A}-\boldsymbol{C})$ show Alcam expression in dopaminergic and nondopaminergic neurons. Nondopaminergic neuronal populations display a range of expression from very strong, to completely absent. In dopaminergic neurons, punctate Alcam expression was present in cell bodies and axons $(\boldsymbol{D})$ and in $\mathrm{F}$-actin ${ }^{+}$growth cones $(\boldsymbol{E})$. Representative images and corresponding axonal traces of $\mathrm{TH}^{+}$neurons in primary midbrain cultures grown on control $(\boldsymbol{F})$, or high-density Alcam substrate $(\boldsymbol{G})$. Quantification of axon growth in the presence of an Alcam substrate resulted in a significant increase in length of the longest (dominant) neurite $(\boldsymbol{H})$ (unpaired $t$ test, $t=4.533, \mathrm{df}=8, p=0.0019, n=5$ ) and the length of all (total) neurites per neuron (I) (unpaired $t$ test, $t=2.659, \mathrm{df}=8, p=0.0289, n=$ 5). No significant effect was observed on number of branches $(\boldsymbol{J})$ or number of neurites $(\boldsymbol{K})$ per neuron. Representative traces of nondopaminergic midbrain neuron traces (Tuj $\left.1^{+} / \mathrm{TH}^{-}\right)$from primary midbrain neurons cultured on control $(\boldsymbol{L})$ or high-density Alcam substrate $(\boldsymbol{M})$. Quantification of axon growth in the presence of Alcam substrate resulted in no significant effect on neurite length $(\boldsymbol{N})$ or branch number $(\boldsymbol{O})(n=5)$. Representative axonal traces of dopaminergic $\left(\mathrm{TH}^{+}\right)$neurons in primary midbrain cultures from WT $(\boldsymbol{P})$ and Alcam $^{-1-}$ mice $(\mathbf{Q})$. Quantification of axon growth showed no significant changes due to loss of Alcam function on (R) neurite length or (S) branch number (unpaired t test, $n=4$ ). Scale bars: $\boldsymbol{A}-\boldsymbol{D}, 20 \mu \mathrm{m} ; \boldsymbol{E}, 10 \mu \mathrm{m} ; \boldsymbol{F}, \boldsymbol{G}, \boldsymbol{L}, \boldsymbol{M}, \boldsymbol{P}, \boldsymbol{Q}, 20 \mu \mathrm{m}$. Data are shown as mean \pm SEM. ${ }^{* *} p<0.01,{ }^{* * *} p<0.001$.

with significantly increased neurite length observed in both WT $(55.7 \pm 15.9 \%)$ and Alcam $^{-1-}(45.4 \pm 13.9 \%)$ neurons (Fig. $4 C$ ), suggesting that Alcam was not functioning through transhomophilic interactions. No significant effect was observed on branch number (Fig. 4D).

\section{Alcam substrate promotes axon growth through L1 family CAMs}

We next explored the possibility of a heterophilic interaction. In the brain, L1cam is the only known Alcam heterophilic binding partner (DeBernardo and Chang, 1996; Buhusi et al., 2009) and, interestingly, L1-null mice have previously been reported to show abnormal targeting of dopaminergic nigral neurons (Demyanenko et al., 2001). This led us to examine the potential for L1cam on $\mathrm{mDA}$ axons to bind Alcam substrate in trans to facilitate the observed regulation of axon growth. We also examined Chll in the same context, a structurally and functionally related member of the L1cam family with high homology to L1cam that we have previously reported as being expressed in $\mathrm{mDA}$ neurons and contributing to the development of mDA pathways (Bye et al., 2015; Alsanie et al., 2017).

Immunohistochemical analysis of E12.5 mouse brain showed that both L1cam and Chll were expressed throughout the developing $\mathrm{mDA}$ pathway during the peak period of $\mathrm{mDA}$ axon growth (Fig. 5A). Both proteins were robustly expressed in the ventral midbrain, predominately within the mantle zone where the soma of mDA neurons reside (Fig. $5 B-D$ ), as well as in the developing medial forebrain bundle (Fig. $5 E-G$ ) and ganglionic eminence (Fig. $5 H-J$ ). High-magnification images showed that L1cam and Chl1 were expressed on both $\mathrm{TH}^{+}$mDA axons and also non-mDA fibers in these areas (Fig. $5 K-P$ ).

Immunocytochemistry in primary $\mathrm{VM}$ cultures enabled a more detailed assessment of the cellular pattern of expression of L1cam and Chl1. Both CAMs were expressed in midbrain neurons, with significant variation in the ratio of expression in nondopaminergic neurons (Fig. 6A). L1cam (Fig. 6B,C) and Chl1 (Fig. $6 D, E$ ) were ubiquitously expressed in the cell body, neurites, branches, and growth cones in all neurons. Highermagnification images in dopaminergic neurons show evenly distributed punctuate expression throughout the neuron, including the growth cone for both L1cam (Fig. $6 F-H$ ) and Chl1 (Fig. $6 I-K)$. Neutralizing antibodies for L1cam or Chll were used to assess their functional role in $\mathrm{mDA}$ neurons as trans-heterophilic binding partners for the growth-promoting effect of substratebound Alcam. Primary ventral midbrain neurons were grown on wells coated with control or Alcam substrate in the presence of an IgG control or neutralizing antibodies. For L1cam, two neutralizing antibodies were tested in parallel, the previously reported 6096 L1-blocking antibody (Thelen et al., 2012) and a commercially available $\alpha$-L1cam (R\&D Systems clone 324, data not shown). Both L1 antibodies nearly completely abolished the growth-promoting effect of the Alcam substrate, from $51.2 \pm$ 

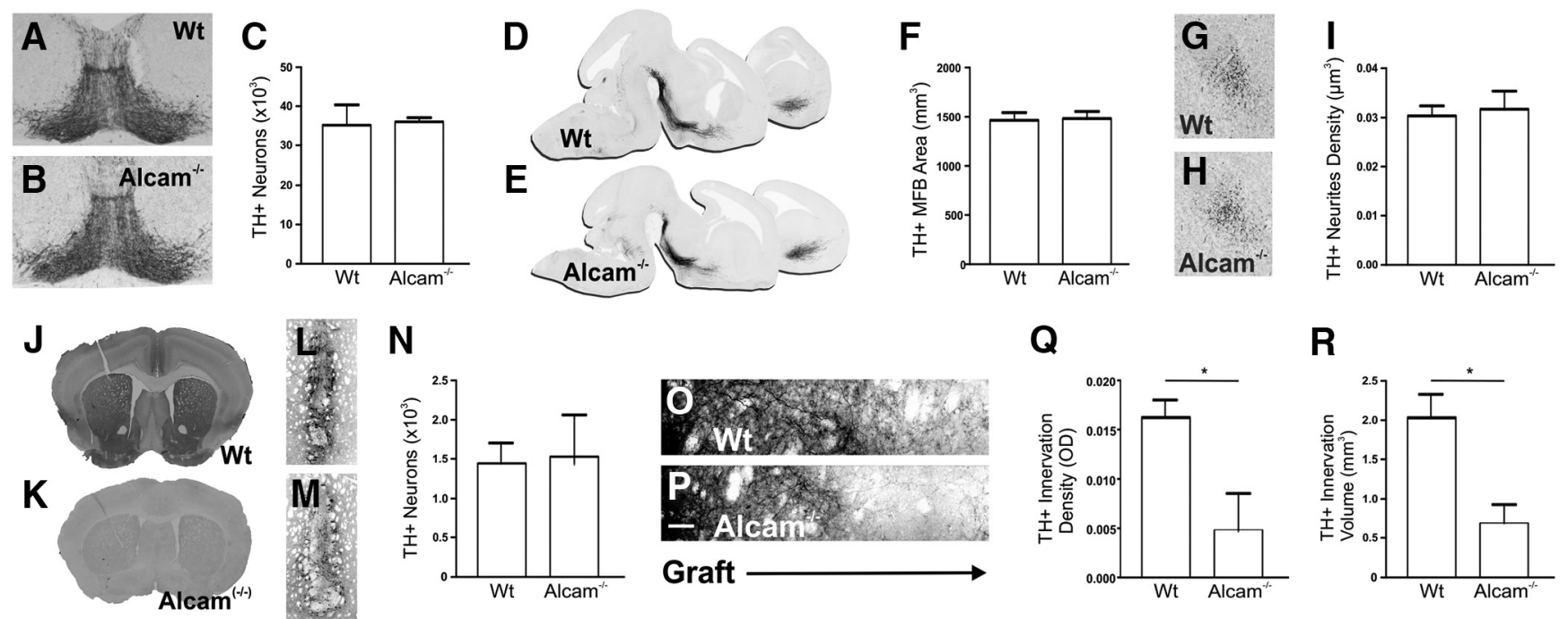

Figure 3. Alcam loss of function in midbrain dopamine neurons during axonal pathway formation in vivo. Representative coronal images of the midbrain at E14.5 in WT $(\boldsymbol{A})$ and Alcam ${ }^{-1-}(\boldsymbol{B})$ mice showed no significant difference in immunolabeled TH neuron number $(\boldsymbol{C}$ (unpaired $t$ test, $n=4$ ). Sagittal images of the developing midbrain dopamine axonal pathway at E14.5 in WT (D) and Alcam ${ }^{-1-}(\boldsymbol{E})$ mice immunolabeled for TH revealed no gross defect in pathway growth or trajectory. Quantification of total volume $(\boldsymbol{F})$ of the axon bundle, or in the density of dopaminergic varicosities $(\mathbf{G}-\boldsymbol{I})$ reaching the forebrain revealed no significant difference in axon fasciculation or pathfinding (unpaired $t$ test, $n=4$ ). Coronal images of adult mouse forebrain immunolabeled for Alcam show extensive expression throughout the striatum of WT mice $(J)$, but completely absent in Alcam ${ }^{-1-}$ mice $(\boldsymbol{K})$. Six weeks after transplantation of dissociated E12.5 ventral midbrain neurons into the striatum of WT $(\boldsymbol{L})$ or Alcam $^{-1-}$ mice $(\boldsymbol{M})$, coronal images show no significant difference in TH immunolabeled neuron survival $(\boldsymbol{N})$ (unpaired $t$ test, $\left.n=4\right)$. Magnified images immediately adjacent to the transplanted neuron graft $(\boldsymbol{O}, \boldsymbol{P})$ show a significant reduction of $0.0113 \pm 0.0048$ in the density of $\mathrm{TH}$ innervation $(\boldsymbol{Q})$ (unpaired $t$ test $, t=2.340, \mathrm{df}=8, p=0.0474$, $n=4)$ in the Alcam ${ }^{-1-}$ host. Quantification of axonal innervation volume from WT neurons into an Alcam ${ }^{-1-}$ host resulted in a significant reduction of $1.342 \pm 0.3782 \mathrm{~mm}^{3}$ in the volume of innervation $(\boldsymbol{R})$ (unpaired $t$ test, $t=2.538 \mathrm{df}=8, p=0.0075, n=4$ ). Scale bars in $\boldsymbol{O}$ and $\boldsymbol{P}, 10 \mu \mathrm{m}$. Data are shown as mean \pm SEM. ${ }^{*} p<0.05$.

A

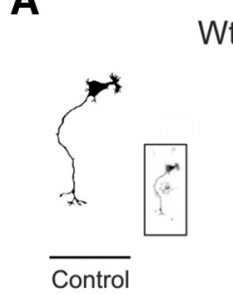

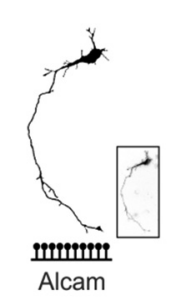
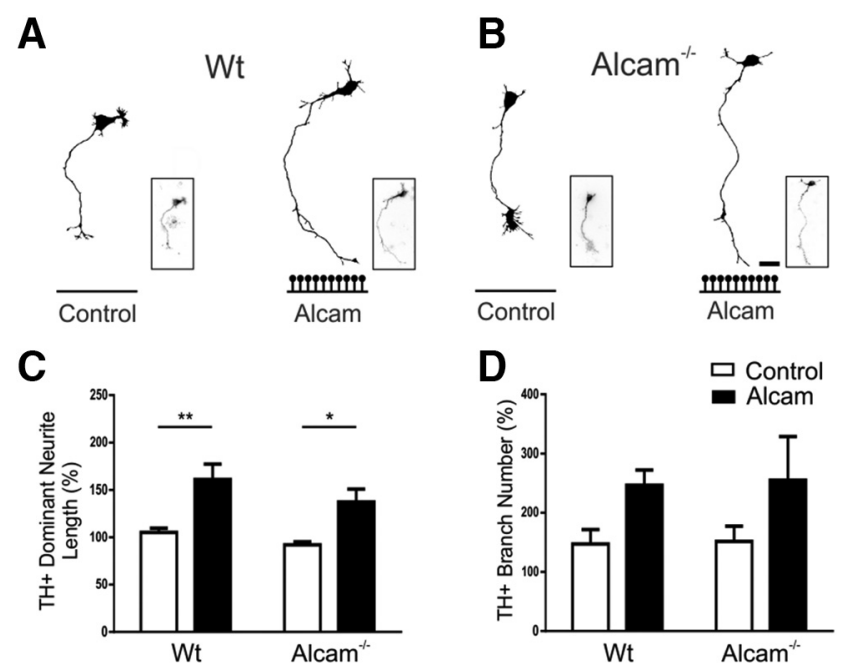

Figure 4. Functional analysis of Alcam homophilic and trans-heterophilic interactions in the promotion of axon growth by an Alcam substrate. Representative $\mathrm{TH}^{+}$axonal traces from primary ventral midbrain cultures of WT $(\boldsymbol{A})$ or Alcam $^{-1-}(\boldsymbol{B})$ neurons cultured on control or Alcam substrate. Quantification of axon growth in the presence of an Alcam substrate resulted in a significant increase in neurite length $(\boldsymbol{C})$ (two-way ANOVA, Alcam substrate, $F_{(1,12)}=$ $23.05, p=0.004, n=4)$ in both WT $(t=3.741, \mathrm{df}=12, p=0.0056)$ and Alcam $^{-1-}$ neurons ( $t=3.049, \mathrm{df}=12, p=0.0201$ ), where loss of Alcam function on growing axons prevents homophilic interactions. A significant effect on branch number $(\boldsymbol{D})$ (two-way ANOVA, substrate, $F_{(1,12)}=5.554, p=0.0363, n=4$ ) was not significant in post hoc tests. Scale bars in $\boldsymbol{A}$ and $\boldsymbol{B}$, $20 \mu \mathrm{m}$. Data are shown as mean \pm SEM. ${ }^{*} p<0.05,{ }^{* *} p<0.01$.

$10.3 \%$ to $7.8 \pm 9.8 \%$ (Fig. $6 L-N$ ). No significant effect was observed on branch number (Fig. 6O). Incubation with the Chl1neutralizing antibody produced similar results, with the previously observed increase in $\mathrm{mDA}$ neurite growth on Alcam substrate $(78.6 \pm 25.0 \%)$ again almost completely abolished

$(10.3 \pm 14.3 \%)$ (Fig. $6 P-R)$. No significant effect was observed on branch number (Fig. $6 S$ ).

Although L1 cam-Alcam interactions have been described previously (DeBernardo and Chang, 1996; Buhusi et al., 2009), Chl1 is not a known Alcam binding partner. To determine the possibility of a direct Alcam-Chl1 interaction, we used an in situ PLAlabeling assay in which target proteins colocated within $40 \mathrm{~nm}$ yield a fluorescent signal. In primary midbrain cultures immunolabeled with Alcam, Chl1, and TH, fluorescent Alcam-Chl1 interactions were quantified on the growth cone of actively growing dominant mDA neurites from WT and Alcam ${ }^{-/-}$neurons grown on control or Alcam substrate (Fig. 6T-Z). Compared with Alcam ${ }^{-1-}$ neurons grown on control substrate (no cis or trans interactions; Fig. 6T,U), neurons cultured on Alcam substrate from WT mice (both cis and trans interactions possible; Fig. $6 \mathrm{~V}, W)$ resulted in a 49 -fold increase in positive puncta, whereas neurons cultured on Alcam substrate from Alcam ${ }^{-1-}$ mice (trans interactions only; Fig. $6 X, Y$ ) increased by 20 -fold (Fig. 6Z). Puncta not located on $\mathrm{TH}^{+}$mDA growth cones (resulting from Alcam-Chl1 interactions between non-mDA axons) were excluded from analysis. Overall, $40 \%$ of all puncta quantified in the PLA assays were attributable to a trans-heterophilic interaction between Chl1 on the growth cone and plated Alcam substrate.

\section{Alcam functionally interacts with the semaphorin receptor complex to promote mDA axon growth}

Previous studies have shown that both L1cam and Chl1 can functionally interact with the Neuropilin 1 (Nrp1) semaphorin receptor complex (Schlatter et al., 2008; Demyanenko et al., 2011) and led us to hypothesize that the same binding complex may be involved in Alcam modulation of $\mathrm{mDA}$ neurite growth. In primary midbrain cultures, Nrp1 and Nrp2 were expressed in the cell body, axons, branches, and growth cones of all neurons, with a distinct variation in the ratio of expression of the two receptors in nondopaminergic neurons (Fig. 7A). In dopaminergic neu- 
A
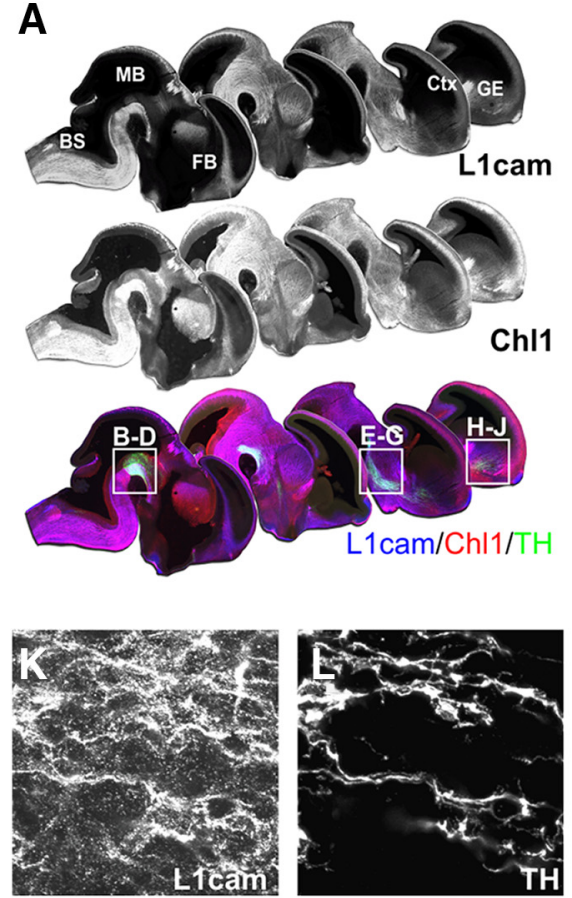
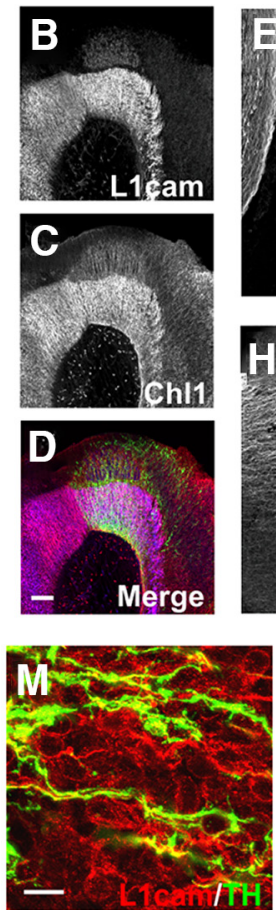
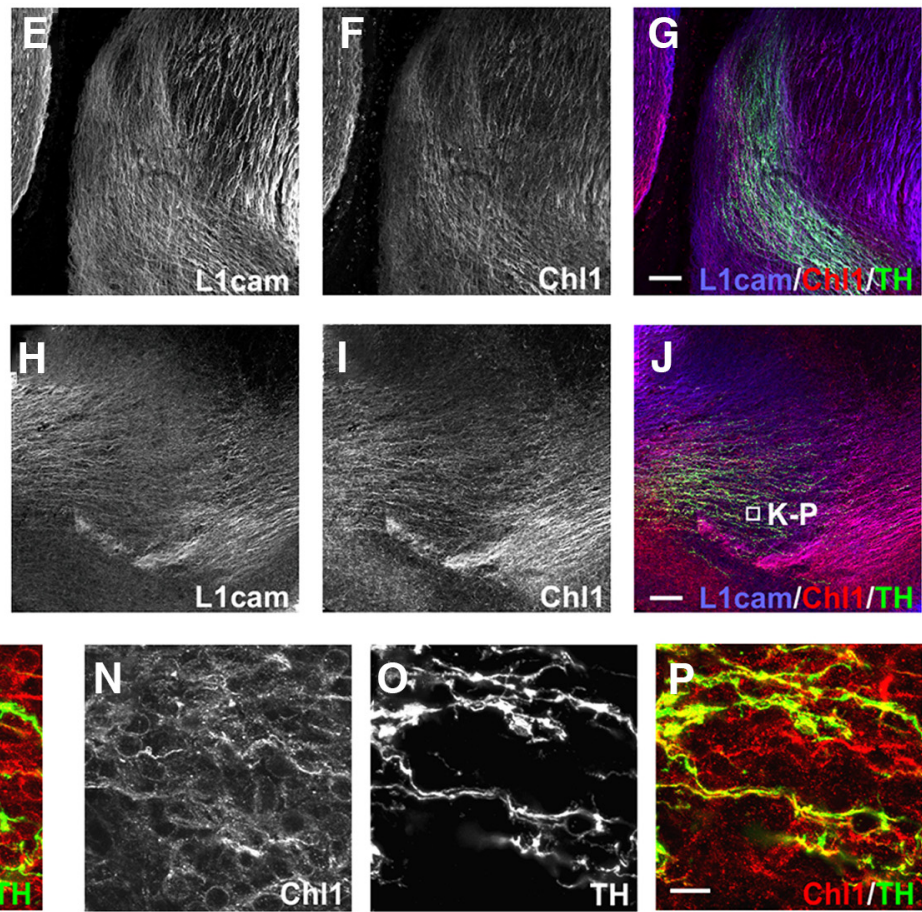

Figure 5. Expression of L1cam and Chl1 in vivo during formation of the midbrain dopaminergic axonal pathways. Sagittal images of whole embryonic brain at E12.5 (A) immunolabeled for L1 cam, Chl1, and L1cam/Chl1/TH showing their expression in the developing dopaminergic pathway (displayed in a medial to lateral series). Inserts show both L1cam and Chl1 were highly expressed in the ventral midbrain $(\boldsymbol{B}-\boldsymbol{D})$, including in dopaminergic neurons along the mDA axonal pathway $(\boldsymbol{E}-\boldsymbol{G})$ in both dopaminergic and nondopaminergic axons. In the forebrain $(\boldsymbol{H}-\boldsymbol{J})$, L1cam and $\mathbf{C h l 1}$ expression was located in both dopaminergic axons and nondopaminergic axons, with prominent expression in the cortex. Magnified images show colocalization maps of $\mathrm{L} 1 \mathrm{cam}$ ( $\boldsymbol{K}-\boldsymbol{M}$ ) or $\mathrm{Chl1}$ $(\boldsymbol{N}-\boldsymbol{P})$ in dopaminergic axons and growth cones at the axonal front. Scale bars: $\boldsymbol{B}-\boldsymbol{J}, 20 \mu \mathrm{m} ; \boldsymbol{K}-\boldsymbol{P}, 10 \mu \mathrm{m}$. BS, Brainstem; Ctx, cortex; FB, forebrain; GE, ganglionic eminence; MB, midbrain.

rons, both Nrp1 and Nrp2 were highly and ubiquitously expressed throughout the neuron (Fig. $7 B-D$ ), including in the growth cone (Fig. $7 E$ ). In culture, an increase in $\mathrm{mDA}$ neurite growth when plated on Alcam substrate $(48.3 \pm 4.6 \%)$ was almost completely abolished in the presence of a neutralizing antibody for Nrp1 $(2.2 \pm 8.3 \%)$, but not with an Nrp2-neutralizing antibody $(31.0 \pm 9.2 \%$, Fig. $7 F-I)$. No significant effect was observed on branch number (Fig. $7 J$ ). To further substantiate the finding of a novel functional interaction between Nrpl and Alcam, midbrain primary cultures were immunolabeled with Alcam, Nrp1, and TH, and direct Alcam-Nrp1 interactions identified using the in situ PLA-labeling assay (Fig. $7 \mathrm{~K}-\mathrm{Q}$ ). Relative to Alcam $^{-1-}$ neurons grown on a control substrate (no cis or trans interactions) (Fig. $7 K, L$ ), WT neurons cultured on Alcam substrate (both cis and trans interactions possible) resulted in a 41fold increase in puncta (Fig. $7 \mathrm{M}, N$ ), whereas Alcam $^{-1-}$ neurons cultured on Alcam substrate (trans interactions only, Fig. 7O,P) increased by 24 -fold (Fig. 7Q). Overall, $58 \%$ of all puncta quantified in the PLA assays were attributable to a trans-heterophilic interaction between Nrp1 on the growth cone and plated Alcam substrate.

\section{Signaling by soluble semaphorins is modulated in trans by Alcam}

The involvement of the semaphorin Nrp1 receptor in mediating the effect of Alcam on mDA axon growth suggests an interaction between substrate bound Alcam and soluble semaphorins. In the context of mDA neurite growth, the class 3 semaphorins Sema3A, Sema3C, and Sema3F have previously been implicated in axon growth and guidance (Hernández-Montiel et al., 2008; Kolk et al., 2009; Tamariz et al., 2010). Sema3A acts via the Nrp1 receptor, Sema3C through both Nrp1 and Nrp2 receptors, and Sema3F through the Nrp2 receptor (Yazdani and Terman, 2006). To determine whether Alcam substrate modulated soluble semaphorin signaling, primary ventral midbrain neurons were grown on wells coated with control or Alcam substrate and treated with soluble Sema3A, Sema3C, or Sema3F (Fig. $8 A-D$ ). In untreated control neurons, Alcam substrate induced a significant increase in neurite length of mDA neurons $(49.6 \pm 2.7 \%)$. In agreement with previous observations (Hernández-Montiel et al., 2008), neurite length of mDA neurons treated with Sema3A and grown on control substrate was increased $(50.7 \pm 12.8 \%)$, an effect lost in Sema3A treated neurons grown on Alcam substrate $(0.26 \pm$ $4.5 \%)$. Alcam substrate had no effect on Sema3C or Sema3F treatment, with neurite length increased by Alcam substrate in Sema3C- $(50.6 \pm 7.8 \%)$ and Sema3F (28.9 $\pm 7.9 \%)$-treated cultures (Fig. 8E). No significant effect of Alcam substrate was observed on branch number in control, Sema3A-, and Sema3Ftreated mDA neurons. However, Sema3C-treated neurons grown on Alcam, but not the control, substrate resulted in significantly enhanced branching $(186.9 \pm 21.5 \%$, Fig. $8 F)$.

To determine whether the modulation of Semaphorin signaling by Alcam substrate was specific to $\mathrm{mDA}$ neurons, non-mDA neurons $\left(\mathrm{TH}^{-} / \mathrm{Tuj}^{+}\right)$in the midbrain primary cultures were examined using the same parameters. No significant change in neurite length (Fig. $8 G$ ) was observed due to Semaphorin treatment in the presence or absence of Alcam substrate. In contrast, branch number in non-mDA neurons was increased by Sema3A treatment $(188.92 \pm 17.70 \%)$, an effect reversed in the presence of Alcam substrate $(88.13 \pm 26.68 \%$, Fig. $8 H)$. The proposed interactions that allow for context-specific modulation of $\mathrm{mDA}$ growth by semaphorins based on the presence or absence of Alcam are summarised in Fig. $8 I, J$. 

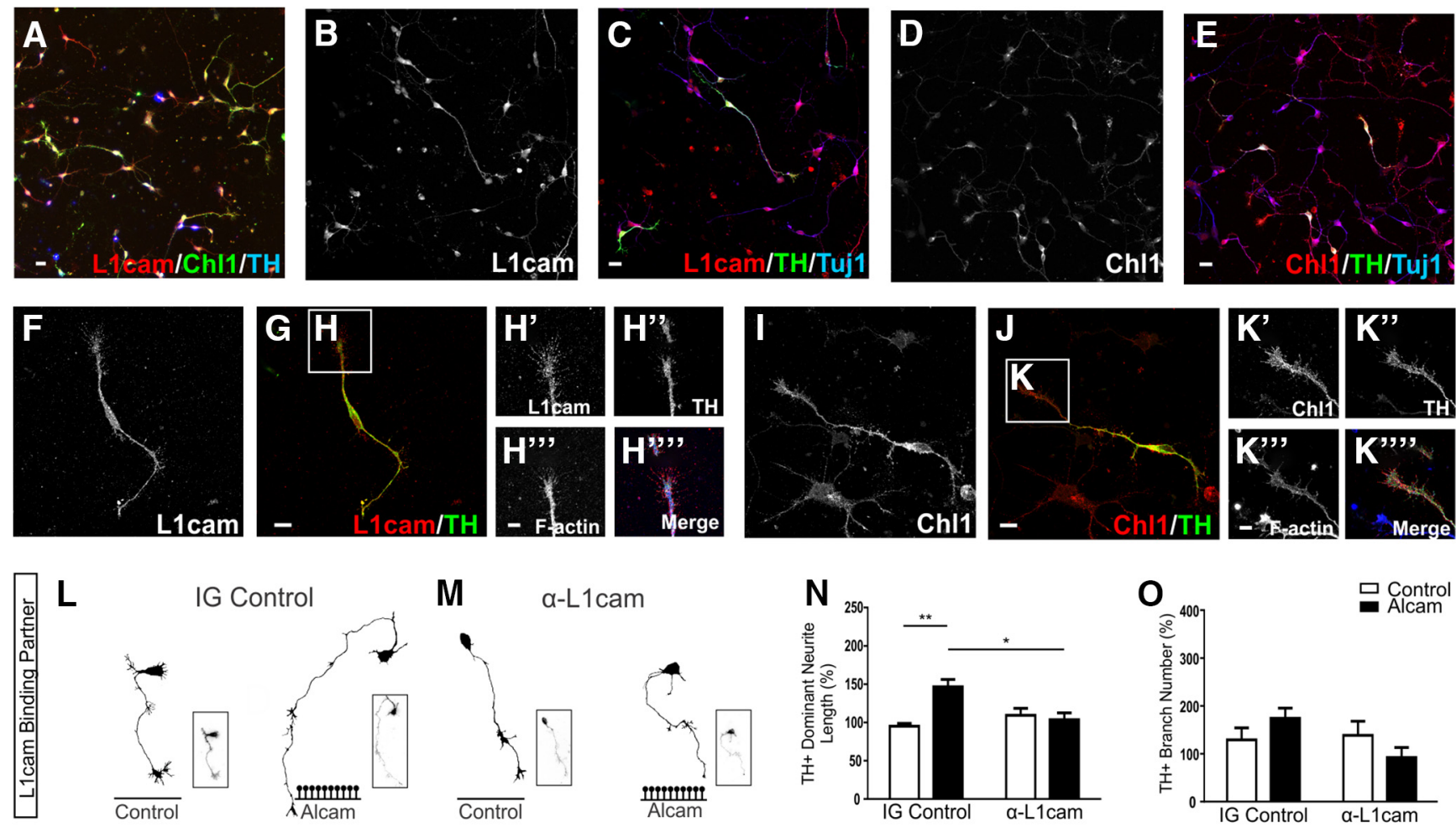

M $\quad \alpha-L 1$ cam
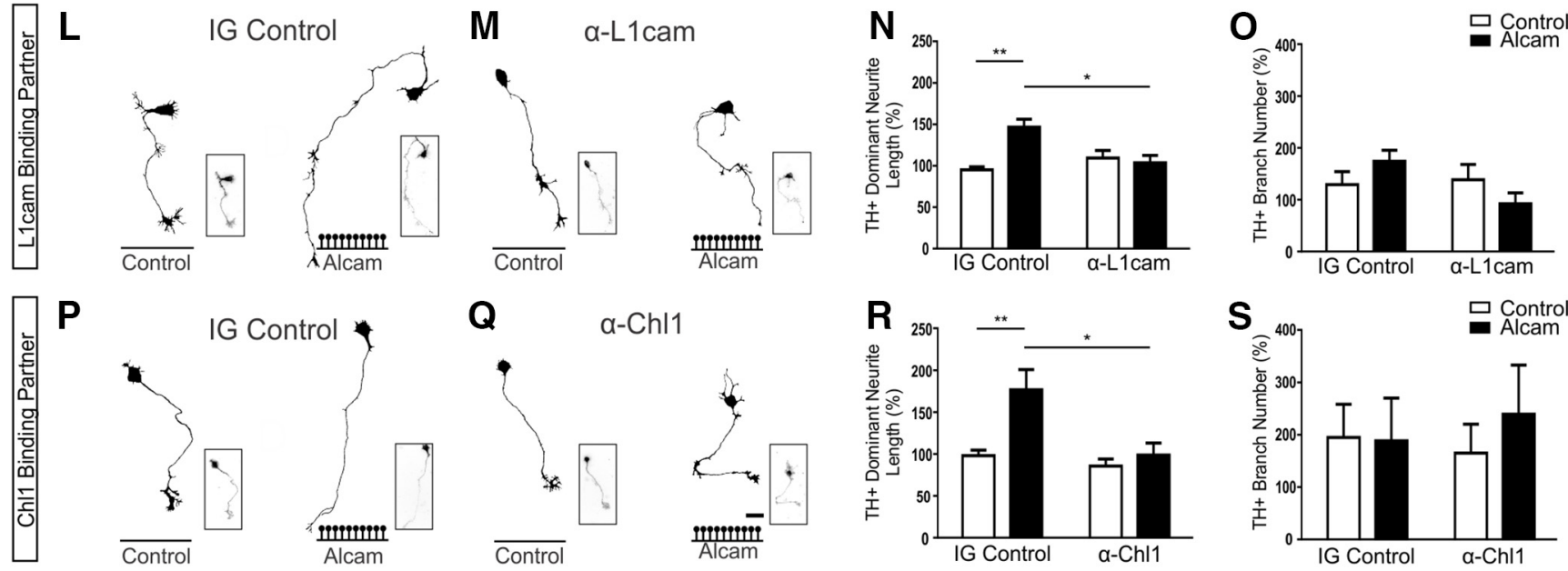

Q $\quad \alpha-C h \mid 1$
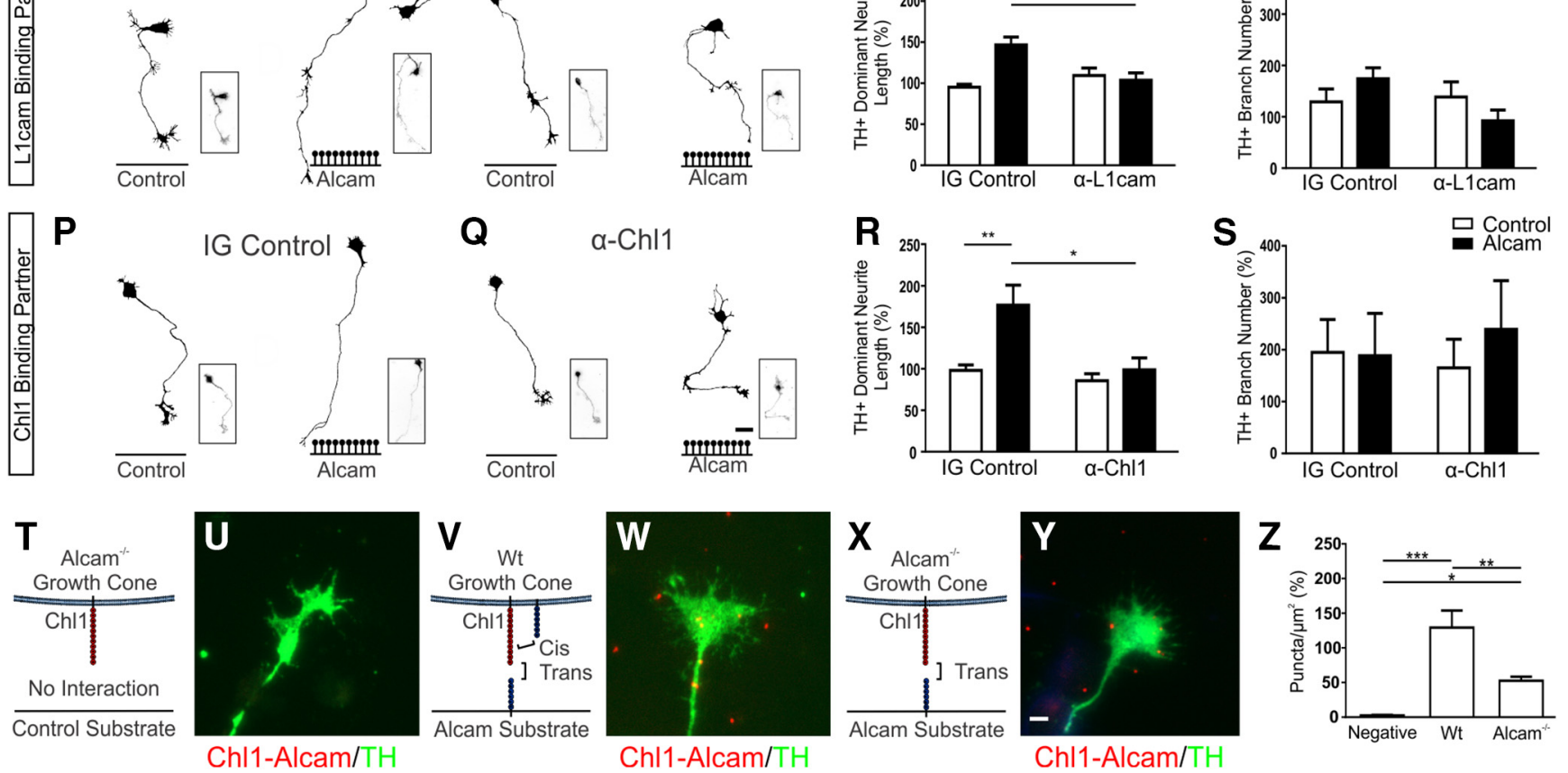

Figure 6. Functional analysis of Alcam trans-heterophilic interactions with the potential binding partners L1cam and Chl1. Representative images of primary midbrain cultures immunolabeled for the potential Alcam trans-heterophilic binding partners L1cam and Chl1 show broad expression of both CAM's in dopaminergic and nondopaminergic neurons $(\boldsymbol{A})$. Expression was localized throughout the cell body, axons, and branches of all neurons for $L 1 \mathrm{cam}(\boldsymbol{B}, \boldsymbol{C})$, and $C h \mid 1(\boldsymbol{D}, \boldsymbol{E})$. In dopaminergic neurons, L1cam expression was evenly distributed in cell bodies, axons $(\boldsymbol{F}, \boldsymbol{G})$, and F-actin ${ }^{+}$growth cones $(\boldsymbol{H})$. Parallel images of the potential Alcam-binding partner $\mathrm{Chl}(\boldsymbol{I}, \boldsymbol{J})$ also show $\mathrm{Chl1}$ expression in cell bodies, axons, and F-actin ${ }^{+}$growth cones $(\boldsymbol{K})$. Representative dopaminergic $\left(\mathrm{TH}^{+}\right)$neuron traces of WT neurons cultured on control or Alcam substrate in the presence of an I $G$ control $(\boldsymbol{L})$ or L1cam-neutralizing antibody $(\boldsymbol{M})$, blocking potential trans heterophilic interactions between Alcam and L1cam. A significant interaction (two-way ANOVA, $\left.F_{(1,8)}=9.264, p=0.0160\right)$ blocking antibody $\left(F_{(1,8)}=6.531, p=0.0339\right)$ and substrate $\left(F_{(1,8)}=17.18, p=\right.$ $0.0032)$ effect was detected $(n=3)$. The significant growth promoting effect of Alcam substrate on neurite length $(\boldsymbol{N})(t=5.083, \mathrm{df}=8, p=0.0019)$ was ablated in the presence of functional blocking of $\mathrm{L} 1 \mathrm{cam}(t=0.7783, \mathrm{df}=8, p=0.7071)$. Neurite length decreased significantly in a direct comparison of neurons grown on Alcam substrate in control and in the presence of the $\mathrm{L} 1 \mathrm{cam}$ blocking antibody ( $t=3.959, \mathrm{df}=8, p=0.0248)$. No significant effect on branch number $(\boldsymbol{O})$ was observed. Representative dopaminergic neuron traces of WT neurons cultured on control or Alcam substrate in the presence of $\mathrm{I}$ control $(\boldsymbol{P})$ or Chl1-neutralizing antibody $(\boldsymbol{Q})$, blocking potential trans heterophilic interactions between Alcam and Chl1. A significant interaction (two-way ANOVA, $\left.F_{(1,8)}=5.650, p=0.0447\right)$ blocking antibody $\left(F_{(1,8)}=10.52, p=0.0118\right)$ and substrate $\left(F_{(1,8)}=9.549, p=0.0149\right)$ effect was detected $(n=3)$. The significant growth promoting effect of Alcam substrate on neurite length $(R)(t=3.866, \mathrm{df}=8, p=0.0095)$ was ablated in the presence of functional blocking of $\mathrm{Chl} 1(t=0.5042, \mathrm{df}=8, p=0.8614)$. Neurite length decreased significantly in a direct comparison of neurons grown on Alcam substrate in control and in the presence of the Chl1 blocking antibody $(t=3.975, \mathrm{df}=8, p=0.0243)$. No significant effect on branch number (S) was observed. Representative immunohistochemistry images of primary dopaminergic growth cones immunolabeled using an in situ proximity ligation assay to detect interactions between Alcam and Chl1. Puncta (shown in red) indicate positive Alcam-Chl1 interactions in Alcam ${ }^{-1-}$ neurons grown on a control substrate ( $\boldsymbol{T}, \boldsymbol{U}$, no cis or trans interactions), WT neurons cultured on Alcam substrate ( $\boldsymbol{V}, \boldsymbol{W}$, cis and trans interactions) and Alcam ${ }^{-1-}$ neurons grown on an Alcam substrate $\left(\boldsymbol{X}, \boldsymbol{Y}\right.$, trans interactions only). Quantification of Alcam-Chl1 ${ }^{+}$puncta $(\boldsymbol{Z})$ showed a significant difference between groups (one-way ANOVA, $F_{(2,8)}=27.00, p=0.0003, n=3-4$ ). Relative to the negative control group, a significant increase in puncta was observed in WT neurons grown on Alcam substrate $(t=10.38, \mathrm{df}=8, p=0.0002)$ and in Alcam ${ }^{-1-}$ neurons grown on Alcam substrate $(t=4.420 \mathrm{df}=8, p=0.0338)$. Comparison of WT and Alcam ${ }^{-1-}$ neurons on Alcam substrate resulted in a significant $40 \%$ reduction in puncta $(t=46.291, \mathrm{df}=8, p=0.0054)$ attributable directly to Alcam-Chl1 trans heterophilic interactions. Scale bars: $\boldsymbol{A}-\boldsymbol{E}, 20 \mu \mathrm{m} ; \boldsymbol{F}, \mathbf{G}, 10 \mu \mathrm{m}$; $\boldsymbol{H}, 5 \mu \mathrm{m} ; \boldsymbol{I}, \boldsymbol{J}, 10 \mu \mathrm{m} ; \boldsymbol{K}, 5 \mu \mathrm{m} ; \boldsymbol{L}, \boldsymbol{M}, \boldsymbol{P}, \boldsymbol{Q}, 20 \mu \mathrm{m} ; \boldsymbol{U}, \boldsymbol{W}, \boldsymbol{Y}, 5 \mu \mathrm{m}$. Data are shown as mean $\pm \mathrm{SEM} .{ }^{*} p<0.05,{ }^{* *} p<0.01,{ }^{* * *} p<0.001$. 

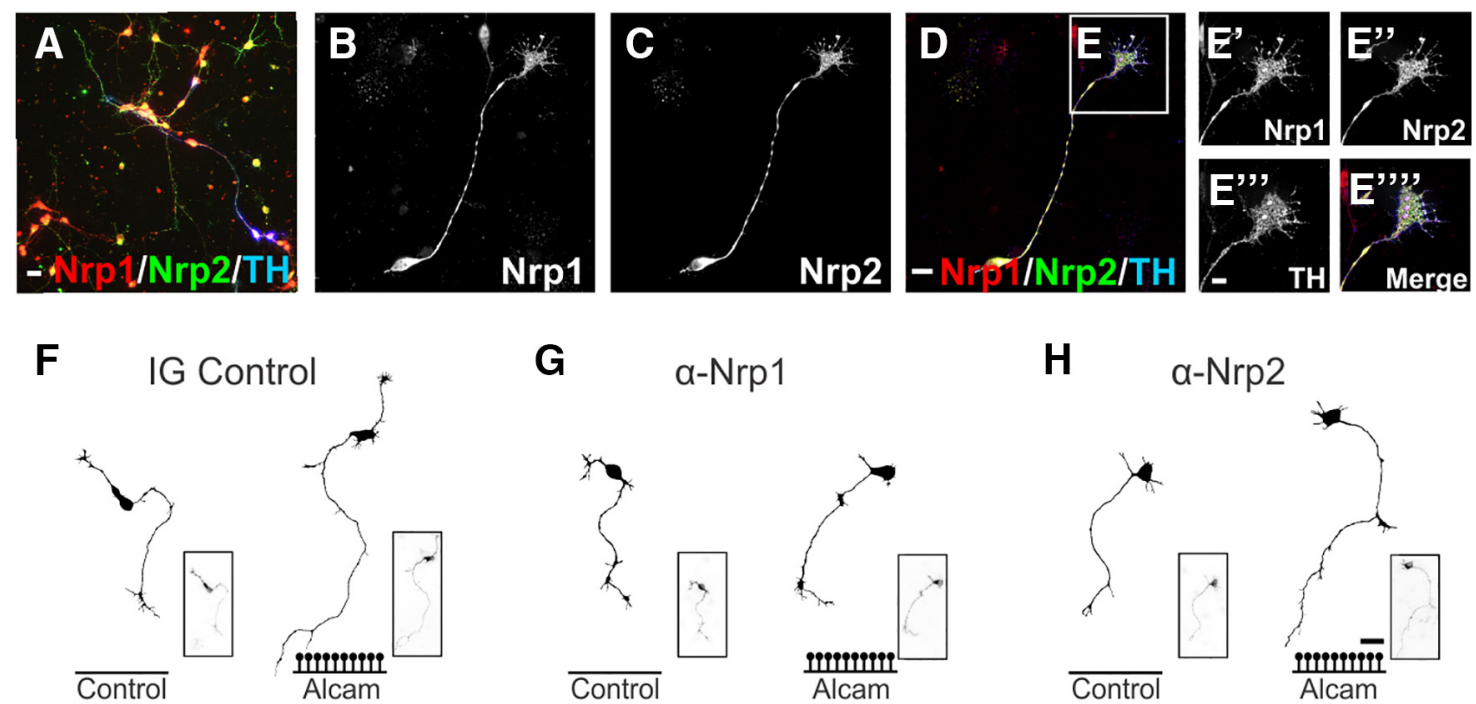

G

a-Nrp1
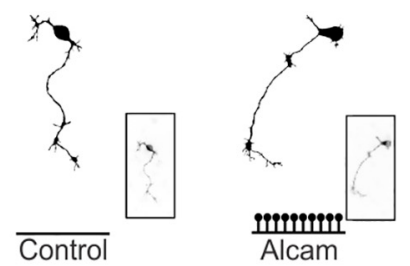

H

$\alpha-\mathrm{Nrp} 2$
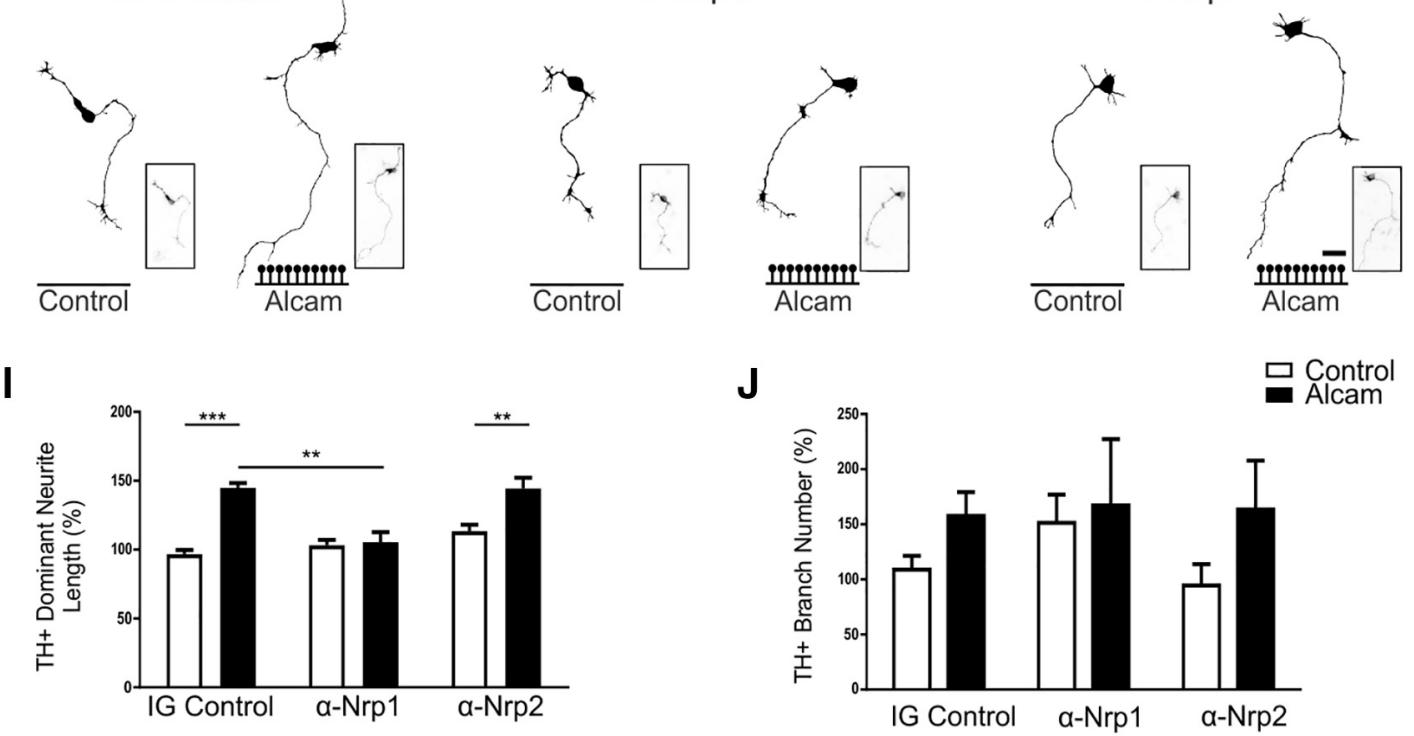

K
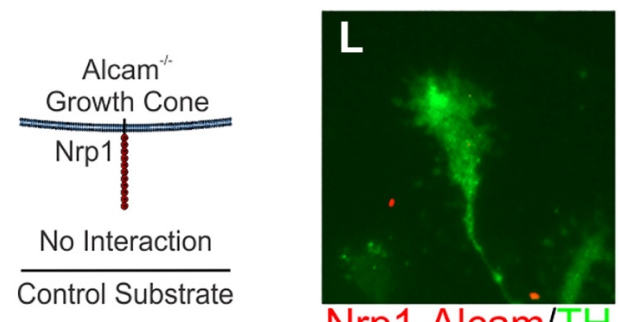

Nrp1-Alcam/TH
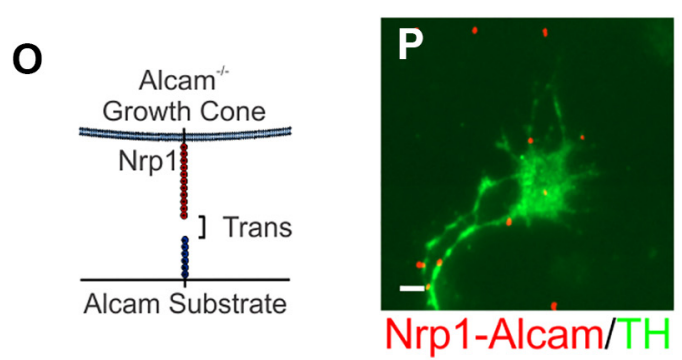

M
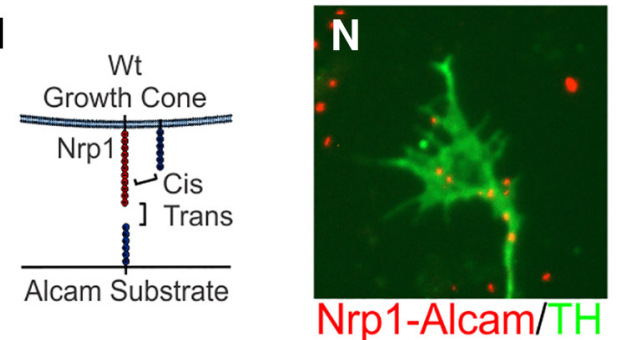

Q

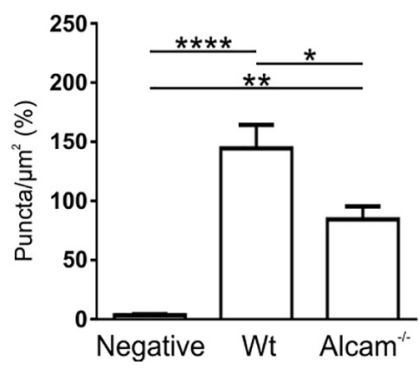

Figure 7. Functional analysis of Alcam trans-heterophilic interactions with the Semaphorin receptors Nrp1 and Nrp2. Representative images of primary midbrain cultures immunolabeled for the potential Alcam trans-heterophilic binding partners Nrp1 and Nrp2 show broad expression of both receptors in dopaminergic and nondopaminergic neurons (A). In dopaminergic neurons, Nrp1 and Nrp2 expression was evenly distributed in cell bodies, axons $(\boldsymbol{B}-\boldsymbol{D})$ and F-actin ${ }^{+}$growth cones $(\boldsymbol{E})$. Representative dopaminergic $\left(\mathrm{TH}^{+}\right)$neuron traces of WT primary midbrain neurons cultured on control or Alcam substrate in the presence of $\mathrm{IG}$ control $(\boldsymbol{F}), \operatorname{Nrp1}(\boldsymbol{G})$, or Nrp2 $(\boldsymbol{H})$-neutralizing antibody, blocking potential trans-heterophilic interactions between Alcam and Nrp1 or Nrp2. Neurite length $(\boldsymbol{I})$ of dopaminergic neurons showed a significant interaction (two-way ANOVA, $\left.F_{(2,12)}=9.385, p=0.0035\right)$ blocking antibody $\left(F_{(2,12)}=10.87, p=0.0020\right)$ and substrate $\left(F_{(1,12)}=38.29, p<0.0001\right)$ effect $(n=3)$. The significant growth promoting effect of Alcam substrate on neurite length $(t=6.354, \mathrm{df}=12, p=0.0001)$ was ablated in the presence of functional blocking of the Nrp1 $(t=0.2888, \mathrm{df}=12, p=0.9890)$, but not Nrp2 receptor ( $t=4.076, \mathrm{df}=12, p=0.0046$ ). No significant effect on $(J)$ branch number was observed (two-way ANOVA, $n=3$ ). Representative immunohistochemistry images of primary dopaminergic growth cones immunolabeled using an in situ proximity ligation assay to detect interactions between Alcam and Nrp1. Puncta (shown in red) indicating positive Alcam-Nrp1 interactions in Alcam ${ }^{-/-}$neurons grown on a control substrate $\left(\boldsymbol{K}, \boldsymbol{L}\right.$, no cis or trans interactions), WT neurons cultured on Alcam substrate $\left(\boldsymbol{M}, \boldsymbol{N}\right.$, cis and trans interactions) and Alcam ${ }^{-/-}$neurons grown on an Alcam substrate $\left(\boldsymbol{O}, \boldsymbol{P}\right.$, trans interactions only). Quantification of Alcam-Nrp ${ }^{+}$puncta $(\boldsymbol{Q})$ showed a significant difference between groups (one-way ANOVA, $\left.F_{(2,9)}=29.10, p=0.0001, n=4\right)$. Relative to the

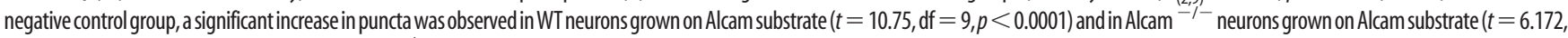
$\mathrm{df}=9, p=0.0046)$. Comparison of WT and Alcam ${ }^{-1-}$ neurons on Alcam substrate resulted in a significant $58 \%$ reduction in puncta $(t=4.577, \mathrm{df}=9, p=0.0250)$ attributable directly to trans heterophilic interactions Scale bars: $\boldsymbol{A}, 20 \mu \mathrm{m} ; \boldsymbol{B}-\boldsymbol{D}, 10 \mu \mathrm{m} ; \boldsymbol{E}, 5 \mu \mathrm{m} ; \boldsymbol{F}-\boldsymbol{H}, 20 \mu \mathrm{m} ; \boldsymbol{L}, \boldsymbol{N}, \boldsymbol{P}, 5 \mu \mathrm{m}$. Data are shown as mean \pm SEM. ${ }^{*} p<0.05,{ }^{* *} p<0.01,{ }^{* * *} p<0.001,{ }^{* * * *} p<0.0001$. 
A

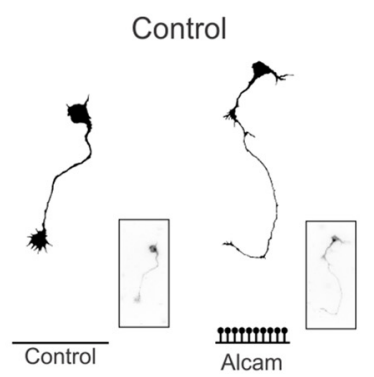

C

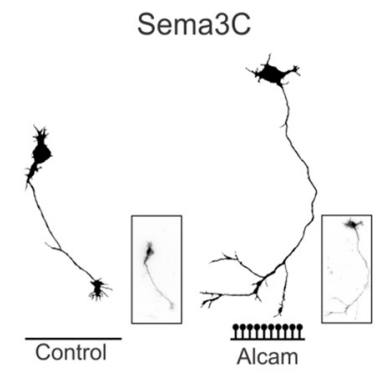

E

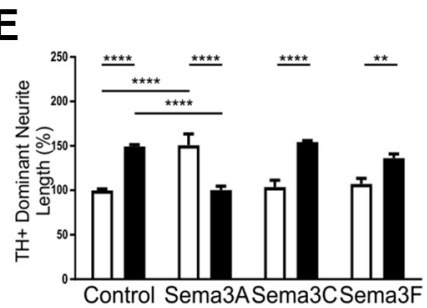

G

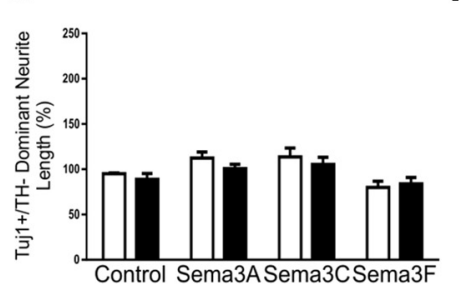

\section{I}

B
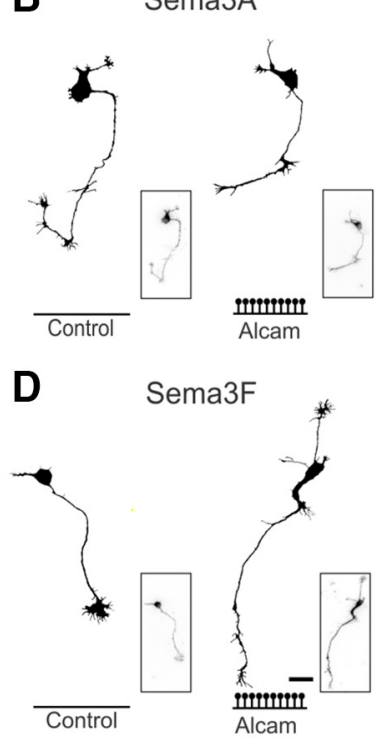

$\mathbf{F}$

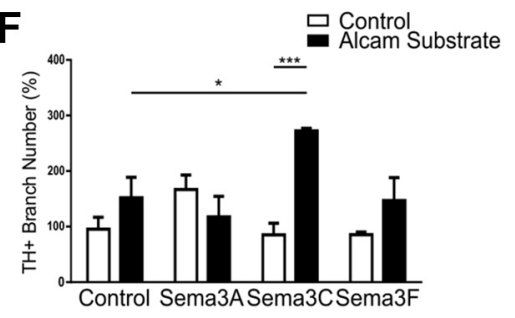

$\mathrm{H}$

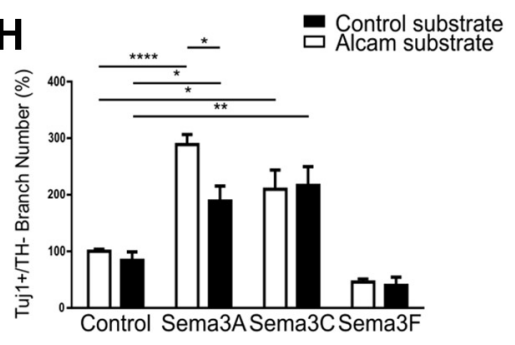

J

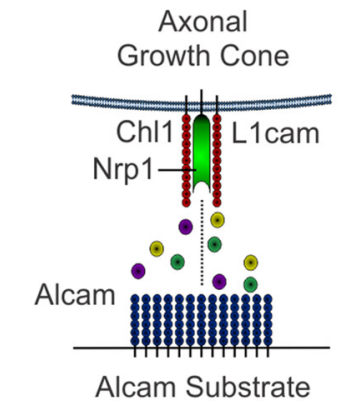

- Sema3A - no effect

- Sema3C - $\uparrow$ Branching

- Sema3F - no effect

Figure 8. Functional analysis of Alcam substrate modulation of Semaphorin signaling. Representative dopaminergic $\left(\mathrm{TH}^{+}\right)$ neuron traces of WT primary midbrain neurons cultured on control or Alcam substrate and treated with control $(\boldsymbol{A})$, Sema3A $(\boldsymbol{B})$, Sema $3 C(\boldsymbol{C})$, or Sema3F $(\boldsymbol{D})$. Neurite length $(\boldsymbol{E})$ of dopaminergic neurons showed a significant interaction (two-way ANOVA, $F_{(3,16)}$ $=28.94, p<0.0001)$ and substrate $\left(F_{(1,16)}=19.59, p=0.0004\right)$ effect $(n=3)$. When grown on Alcam substrate, neurite length was significantly increased in control $(t=5.580, \mathrm{df}=16, p=0.0002)$, significantly decreased with Sema3A treatment $(t=$ $5.677, \mathrm{df}=16, p=0.0001)$, significantly increased with Sema3C treatment $(t=5.697, \mathrm{df}=16, p=0.0001)$, and significantly increased with Sema3F treatment $(t=3.253, \mathrm{df}=16, p=0.0198)$. Direct comparison of Semaphorin-treated neurite length on control substrate significantly increased only with Sema3A treatment $(t=5.729, \mathrm{df}=16, p=0.0009)$. The significant growth promoting effect of Sema3A and Alcam substrate on neurite length was reversed in the presence of both Sema3A and Alcam

\section{Discussion}

In a previous study, we reported that the transmembrane molecules Alcam and Chl1 are expressed by mDA neuroblasts in the ventral midbrain of the developing mouse (Bye et al., 2015). Given the involvement of IgSF-CAMs in the growth and navigation of axons from other systems (for review, see Pollerberg et al., 2013), we were motivated to assess an interaction between these molecules in $\mathrm{mDA}$ circuit formation. The results support a role for substrate-bound Alcam in the developing $\mathrm{mDA}$ pathway as a growthpromoting cue by trans-heterophilic signaling through ChL1 or L1Cam expressed in mDA fibers. Interestingly, this could be modulated by soluble semaphorins in a context-dependent manner based on the class of semaphorin and the mDA or non$\mathrm{mDA}$ identity of neurons in primary cultures.

The capacity for Alcam substrate to promote extension of $\mathrm{mDA}$ neurites in primary VM cultures is consistent with the growth-promoting function of Alcam in other systems, including developing motor neurons as well as retinal and dorsal root ganglia pathways (Ott et al., 2001; Avci et al., 2004; Thelen et al., 2012). The growth-promoting effect showed specificity for mDA neurons and was not a pan-

\section{$\leftarrow$}

substrate $(t=5.529, \mathrm{df}=16, p<0.0013)$. Branch number $(\boldsymbol{F})$ of dopaminergic neurons showed a significant interaction (two-way ANOVA, $F_{(3,16)}=6.166, p=0.0055$ ) and substrate $\left(F_{(1,16)}=10.80, p=0.0046\right)$ effect $(n=3)$. Branch number significantly increased only in dopaminergic neurons grown on an Alcam substrate and treated with Sema3C ( $t=4.808$, $\mathrm{df}=16, p=0.0008)$. Branch number increased significantly in a direct comparison of control and Sema3A-treated neurons grown on Alcam substrate $(t=3.093, \mathrm{df}=16, p=0.0300)$, with no significant effect observed in control and Sema3A or Sema3F treated cultures. In nondopaminergic neurons $\left(\mathrm{TH}^{-} /\right.$ $\mathrm{Tuj}^{+}$), a significant effect on neurite length due to Alcam substrate following Semaphorin treatment $(G)$ (two-way ANOVA, Sema treatment, $\left.F_{(3,16)}=7.213, p=0.0028, n=3\right)$ was not significant in post hoc tests. A significant effect on branch number due to Alcam substrate following Semaphorin treatment $(\boldsymbol{H})$ (two-way ANOVA, Sema treatment, $F_{(3,16)}=$ $37.66, p<0.0001, n=3$ ) was also observed. Sema3A treatment resulted in a significant decrease in branch number $(\boldsymbol{H})$ in nondopaminergic neurons when grown on an Alcam substrate $(t=3.260, \mathrm{df}=16, p=0.0195)$. Branch number increased significantly in a direct comparison of control and Sema3A-treated neurons grown on control substrate $(t=$ $6.142, \mathrm{df}=16, p=0.0004)$, but not in Alcam substrate $(t=$ $3.404, d f=16, p=0.0968)$. Schematic of the regulation of Semaphorin signaling the absence $(\boldsymbol{I})$ or presence $(\boldsymbol{J})$ of Alcam substrate indicating the proposed trans-heterophilic interactions with the semaphorin receptor complex (comprised of Nrp1, L1cam and (Chl1) on the growth cone. Scale bars in $\boldsymbol{A}-\boldsymbol{D}$, $20 \mu \mathrm{m}$. Data are shown as mean \pm SEM. ${ }^{*} p<0.05,{ }^{* *} p<$ $0.01,{ }^{* * *} p<0.001,{ }^{* * * *} p<0.0001$. 
axonal growth signal in primary VM cultures. Alcam has also been shown to facilitate branching in a dose-dependent manner (Thelen et al., 2012) and, although we consistently observed a trend for increased branching in $\mathrm{mDA}$ neurons, this was not significant and may indicate a more modest effect in $\mathrm{mDA}$ neurons or a subthreshold density. The expression of Alcam in both the $\mathrm{TH}^{+} \mathrm{mDA}$ neurons and also throughout the medial forebrain bundle and mesohabenular pathway led us to hypothesize that Alcam functioned through a trans-homophilic interaction, as has been reported for Alcam in other systems including the developing spinal cord (Tanaka et al., 1991; DeBernardo and Chang, 1995; Schubert and Kaprielian, 2001; van Kempen et al., 2001). However, the persistence of the full mDA growthpromoting effect of substrate-bound Alcam when using primary VM cultures from Alcam $^{-1-}$ mice convincingly ruled out a homophilic interaction and suggested a heterophilic signaling mechanism with other transmembrane $\mathrm{mDA}$ proteins. The transient expression of Alcam in early mDA neurons may be associated with functions independent of axonal extension, including cell migration, as reported in other systems (Heffron and Golden, 2000).

Although loss of Alcam has been reported to result in deficits in fasciculation and pathfinding in motor and retinal ganglion cells (Weiner et al., 2004), analysis of the mDA system in embryonic and adult Alcam ${ }^{-1-}$ mouse brain did not reveal a clear deficit in mDA pathway formation. This is not necessarily surprising given the capacity for functional overlap and compensation between axon guidance molecules, including CAMs, where double knock-outs are often required to produce pronounced phenotypes (Uetani et al., 2006; Jeon et al., 2008; Demyanenko et al., 2011; Pollerberg et al., 2013; Levy-Strumpf and Culotti, 2014). In support of a developmentally regulated compensatory mechanism for loss of Alcam, we found that mDA neurons implanted into the fully developed striatum of Alcam ${ }^{-1-}$ mice displayed significantly less axonal outgrowth compared with those implanted to WT mice.

We next explored the possibility that substrate-bound Alcam signals in trans with heterophilic binding partners expressed by $\mathrm{mDA}$ neurons. Currently, the only binding partner described for Alcam in neurons is Llcam, identified in immunoprecipitation assays in chick brain and also as a feature of retinal ganglion neurites showing growth preference for Alcam coated substrates in vitro (DeBernardo and Chang, 1996; Avci et al., 2004) (Buhusi et al., 2009). Chll is a CAM with close homology to L1cam and has previously been shown to cooperate with L1cam to facilitate thalamocortical pathfinding (Demyanenko et al., 2011), is expressed in mDA neuroblasts (Bye et al., 2015), and can regulate $\mathrm{mDA}$ axonal growth and guidance as both a soluble and bound substrate (Alsanie et al., 2017). Both Llcam and Chll were expressed in $\mathrm{mDA}$ neurons, notably including prominent expression in growth cones, and in $\mathrm{TH}^{+}$fibers throughout the major $\mathrm{mDA}$ growth pathways during the peak period of axonal growth. Furthermore, the proximity-ligation assay showed that both proteins were capable of direct binding to Alcam and unlike immunoprecipitation assays, allow for cellular detail to demonstrate binding events at the level of the growth cone. Although AlcamL1cam binding has been shown previously (DeBernardo and Chang, 1996), this is the first reported interaction between Alcam and Chll. Importantly, blocking antibodies for Chl1 or L1cam completely abolished the growth-promoting effect of Alcam in cultured mDA neurons. Collectively, these findings provide a strong case for trans-heterophilic signaling between Alcam and
Chl1 or L1cam as a novel, cell adhesion-based mechanism underlying development of mDA circuitry.

Current models of $\mathrm{mDA}$ growth and navigation are based almost entirely on signaling through soluble growth cues, most notably though the major Semaphorin, Ephrin, Netrin, and Slit families (for comprehensive reviews, see Prasad and Pasterkamp, 2009; Prestoz et al., 2012; Brignani and Pasterkamp, 2017). An important feature of semaphorin signaling is the capacity for the same cue to exert both attractive and repulsive effects on $\mathrm{mDA}$ neurites depending on spatiotemporal context. For example, Sema3F initially acts as a repellent to direct axons away from the midbrain toward the diencephalon, but subsequently attracts axons to cortical targets (Kolk et al., 2009; for review, see Brignani and Pasterkamp, 2017). Interestingly, we found that Alcam could modulate both extension and branching of $\mathrm{mDA}$ neurites in response to soluble Semaphorins, most likely through a complex with Nrp1, Chl1, and/or Llcam. In agreement with previous work (Hernández-Montiel et al., 2008), Sema3A was able to stimulate $\mathrm{mDA}$ axonal growth, but this was completely abolished in the presence of an Alcam substrate. Exposure to Sema3C resulted in a significant increase in branching in the presence of Alcam, but not when Alcam was removed. Thus, the binding status between CAMs on the mDA growth cone to Alcam in the surrounding tissue may provide an anatomically relevant context to determine their response to diffusible semaphorins. This would provide a variable response to the same soluble growth cue based not only on the combination of signaling molecules present on the growth cone, but also on the presence of local adhesion molecules, affording a powerful basis for diversity in pathfinding between different neuronal subtypes. Both Sema3A and 3F are expressed in the GE and deep cortical layers at E14, whereas Sema3C is only expressed at the boundary of the cortical subventricular zone and intermediate zone (Kolk et al., 2009; Andrews et al., 2017). An interaction of Sema3A with Alcam-bound mDA neurites may thus be consistent with modulation of axon growth of nigrostriatal and mesostriatal dopaminergic subpopulations specifically within the ganglionic eminence, whereas distribution of Sema3C may be related to a role in the induction of terminal branching of the mesocortical dopaminergic subpopulation. Such mechanisms may have broad implications for guidance both in dopaminergic neurons and other cell types, and it will be important to determine whether trans interaction with CAM's are involved in the modulation of other soluble guidance cues.

In summary, this study shows that local, contact-mediated signaling between CAMs may be an important and largely unexplored mechanism underlying the growth and guidance of $\mathrm{mDA}$ circuitry, and identifies Alcam-Chll as previously unrecognized trans-heterophilic binding partners. Uniquely, Alcam is also capable of differentially modulating the response of growing axons to soluble semaphorin class 3 family guidance cues through a trans-heterophilic interaction. This novel guidance mechanism provides a means to regulate the response to soluble cues, dependent on the specific CAMs that the growth cone is in contact with at the time of signaling. It will be particularly interesting to explore the involvement of these mechanisms and associated molecules on an mDA neuronal subtype-specific basis as part of ongoing efforts to understand how target acquisition is regulated among mDA subtypes, and how dysregulation might underlie $\mathrm{mDA}$ associated neurodegenerative and psychiatric conditions.

\section{References}

Alsanie WF, Penna V, Schachner M, Thompson LH, Parish CL (2017) Homophilic binding of the neural cell adhesion molecule CHL1 regulates 
development of ventral midbrain dopaminergic pathways. Sci Rep 7: 9368.

Andrews WD, Barber M, Nemitz M, Memi F, Parnavelas JG (2017) Semaphorin3A-neuropilin1 signalling is involved in the generation of cortical interneurons. Brain Struct Funct 222:2217-2233.

Avci HX, Zelina P, Thelen K, Pollerberg GE (2004) Role of cell adhesion molecule DM-GRASP in growth and orientation of retinal ganglion cell axons. Dev Biol 271:291-305.

Brignani S, Pasterkamp RJ (2017) Neuronal subset-specific migration and axonal wiring mechanisms in the developing midbrain dopamine system. Front Neuroanat 11:55.

Blakely BD, Bye CR, Fernando CV, Horne MK, Macheda ML, Stacker SA, Arenas E, Parish CL (2011) Wnt5a regulates midbrain dopaminergic axon growth and guidance. PloS one 6:e18373.

Buhusi M, Demyanenko GP, Jannie KM, Dalal J, Darnell EP, Weiner JA, Maness PF (2009) ALCAM regulates mediolateral retinotopic mapping in the superior colliculus. J Neurosci 29:15630-15641.

Bye CR, Thompson LH, Parish CL (2012) Birth dating of midbrain dopamine neurons identifies A9 enriched tissue for transplantation into parkinsonian mice. Exp Neurol 236:58-68.

Bye CR, Jönsson ME, Björklund A, Parish CL, Thompson LH (2015) Transcriptome analysis reveals transmembrane targets on transplantable midbrain dopamine progenitors. Proc Natl Acad Sci U S A 112:E1946E1955.

DeBernardo AP, Chang S (1995) Native and recombinant DM-GRASP selectively support neurite extension from neurons that express GRASP. Dev Biol 169:65-75.

DeBernardo AP, Chang S (1996) Heterophilic interactions of DM-GRASP: GRASP-NgCAM interactions involved in neurite extension. J Cell Biol 133:657-666.

Demyanenko GP, Shibata Y, Maness PF (2001) Altered distribution of dopaminergic neurons in the brain of L1 null mice. Brain Res Dev Brain Res 126:21-30.

Demyanenko GP, Siesser PF, Wright AG, Brennaman LH, Bartsch U, Schachner M, Maness PF (2011) L1 and CHL1 cooperate in thalamocortical axon targeting. Cereb Cortex 21:401-412.

Evans TA, Bashaw GJ (2010) Axon guidance at the midline: of mice and flies. Curr Opin Neurobiol 20:79-85.

Garel S, López-Bendito G (2014) Inputs from the thalamocortical system on axon pathfinding mechanisms. Curr Opin Neurobiol 27:143-150.

Heffron DS, Golden JA (2000) DM-GRASP is necessary for nonradial cell migration during chick diencephalic development. J Neurosci 20:22872294.

Hernández-Montiel HL, Tamariz E, Sandoval-Minero MT, VarelaEchavarria A (2008) Semaphorins 3A, 3C, and 3F in mesencephalic dopaminergic axon pathfinding. J Comp Neurol 506:387-397.

Herrera E, Erskine L, Morenilla-Palao C (2019) Guidance of retinal axons in mammals. Semin Cell Dev Biol 85:48-59.

Jeon M, Nguyen H, Bahri S, Zinn K (2008) Redundancy and compensation in axon guidance: genetic analysis of the drosophila Ptp10D/Ptp4E receptor tyrosine phosphatase subfamily. Neural Dev 3:3.

Kolk SM, Gunput RA, Tran TS, van den Heuvel DM, Prasad AA, Hellemons AJ, Adolfs Y, Ginty DD, Kolodkin AL, Burbach JP, Smidt MP, Pasterkamp RJ (2009) Semaphorin 3F is a bifunctional guidance cue for dopaminergic axons and controls their fasciculation, channeling, rostral growth, and intracortical targeting. J Neurosci 29:12542-12557.

Levy-Strumpf N, Culotti JG (2014) Netrins and Wnts function redundantly to regulate antero-posterior and dorso-ventral guidance in C. elegans. PLoS Genet 10:e1004381.

Ott H, Diekmann H, Stuermer CA, Bastmeyer M (2001) Function of neurolin (DM-GRASP/SC-1) in guidance of motor axons during zebrafish development. Dev Biol 235:86-97.

Pasterkamp RJ, Kolodkin AL (2013) SnapShot: axon guidance. Cell 153: 494, 494e1-2.

Pollerberg GE, Thelen K, Theiss MO, Hochlehnert BC (2013) The role of cell adhesion molecules for navigating axons: density matters. Mech Dev 130:359-372.

Prasad AA, Pasterkamp RJ (2009) Axon guidance in the dopamine system. Adv Exp Med Biol 651:91-100.

Prestoz L, Jaber M, Gaillard A (2012) Dopaminergic axon guidance: which makes what? Front Cell Neurosci 6:32.

Schlatter MC, Buhusi M, Wright AG, Maness PF (2008) CHL1 promotes Sema3A-induced growth cone collapse and neurite elaboration through a motif required for recruitment of ERM proteins to the plasma membrane. J Neurochem 104:731-744.

Schubert W, Kaprielian Z (2001) Identification and characterization of a cell surface marker for embryonic rat spinal accessory motor neurons. J Comp Neurol 439:368-383.

Tamariz E, Díaz-Martínez NE, Díaz NF, García-Peña CM, Velasco I, VarelaEchavarría A (2010) Axon responses of embryonic stem cell-derived dopaminergic neurons to semaphorins $3 \mathrm{~A}$ and 3C. J Neurosci Res 88 : 971-980.

Tanaka H, Matsui T, Agata A, Tomura M, Kubota I, McFarland KC, Kohr B, Lee A, Phillips HS, Shelton DL (1991) Molecular cloning and expression of a novel adhesion molecule, SC1. Neuron 7:535-545.

Thelen K, Jaehrling S, Spatz JP, Pollerberg GE (2012) Depending on its nano-spacing, ALCAM promotes cell attachment and axon growth. PLoS One 7:e40493.

Thompson LH, Parish CL (2013) Transplantation of fetal midbrain dopamine progenitors into a rodent model of Parkinson's disease. Methods Mol Biol 1059:169-180.

Uetani N, Chagnon MJ, Kennedy TE, Iwakura Y, Tremblay ML (2006) Mammalian motoneuron axon targeting requires receptor protein tyrosine phosphatases sigma and delta. J Neurosci 26:5872-5880.

Van Battum EY, Brignani S, Pasterkamp RJ (2015) Axon guidance proteins in neurological disorders. Lancet Neurol 14:532-546.

Van den Heuvel DM, Pasterkamp RJ (2008) Getting connected in the dopamine system. Prog Neurobiol 85:75-93.

van Kempen LC, Nelissen JM, Degen WG, Torensma R, Weidle UH, Bloemers HP, Figdor CG, Swart GW (2001) Molecular basis for the homophilic activated leukocyte cell adhesion molecule (ALCAM)-ALCAM interaction. J Biol Chem 276:25783-25790.

Wade A, Thomas C, Kalmar B, Terenzio M, Garin J, Greensmith L, Schiavo G (2012) Activated leukocyte cell adhesion molecule modulates neurotrophin signaling. J Neurochem 121:575-586.

Walsh FS, Doherty P (1997) Neural cell adhesion molecules of the immunoglobulin superfamily: role in axon growth and guidance. Annu Rev Cell Dev Biol 13:425-456.

Weiner JA, Koo SJ, Nicolas S, Fraboulet S, Pfaff SL, Pourquié O, Sanes JR (2004) Axon fasciculation defects and retinal dysplasias in mice lacking the immunoglobulin superfamily adhesion molecule BEN/ALCAM/SC1. Mol Cell Neurosci 27:59-69.

Yazdani U, Terman JR (2006) The semaphorins. Genome Biol 7:211. 